\section{Statistical Theory of Passive Location Systems}

DON J. TORRIERI

U.S. Army Countermeasures/Counter-Countermeasures Center

A derivation of the principal algorithms and an analysis of the performance of the two most important passive location systems for stationary transmitters, hyperbolic location systems and directionfinding location systems, are presented. The concentration ellipse, the circular error probability, and the geometric dilution of precision are defined and related to the location-system and received-signal characteristics. Doppler and other passive location systems are briefly discussed.

Manuscript received June 21, 1983.

Author's address: U.S. Department of the Army, Countermeasures/ Counter-Countermeasures Center, 2800 Powder Mill Rd., Adelphi, MD 20783.

0018-9251/84/0300-0183\$1.00 ㄷ 1984 IEEE

\section{INTRODUCTION}

The position of a stationary transmitter or radiating emitter can be estimated from passive measurements of the arrival times, directions of arrival, or Doppler shifts of electromagnetic waves received at various sites. This paper presents a derivation of the principal algorithms and an analysis of the two most important passive location systems for stationary transmitters: hyperbolic location systems and direction-finding location systems [1,2].

Hyperbolic location systems, often called time difference of arrival (TDOA) systems, locate a transmitter by processing signal arrival-time measurements at three or more stations. The measurements at the various stations are sent to a station that is designated the master station and does the processing. The arrival-time measurements at two stations are combined to produce a relative arrival time that, in the absence of noise and interference, restricts the possible transmitter location to a hyperboloid with the two stations as foci. Transmitter location is estimated from the intersections of three or more independently generated hyperboloids determined from at least four stations. If the transmitter and the stations lie in the same plane, location is estimated from the intersections of two or more hyperbolas determined from three or more stations. Fig. 1 illustrates two hyperbolas, each of which has two branches, derived from measurements at three stations. The two hyperbolas have two points of intersection. The resulting location ambiguity may be resolved by using a priori information about the location, bearing measurements at one or more of the stations, or a fourth station to generate an additional hyperbola.

Fig. 2 depicts an aircraft with a direction-finding location system that makes bearing measurements at three different points in its trajectory. The intersection of two bearing lines provides an estimate of the location of the transmitter, which may be on the surface of the Earth or airborne. In the presence of noise, more than two bearing lines will not intersect at a single point. However, the appropriate processing allows an improved estimate of the transmitter position.

The following three sections of this paper present the basic methods of estimation applicable to transmitter location and determine the accuracy of suitable estimators. Sections 5 and 6, respectively, consider passive location systems using arrival-time and bearing measurements. Section 7 summarizes the use of Doppler information. Since the next three sections provide the theoretical framework for the statistical analysis of any passive location system, the reader who is only interested in the applications may wish to omit this material, referring to it as necessary while reading Sections 5-7.

\section{ESTIMATION METHODS}

The components of an $n$-dimensional vector $x$ that is to be estimated are the position coordinates in two or 


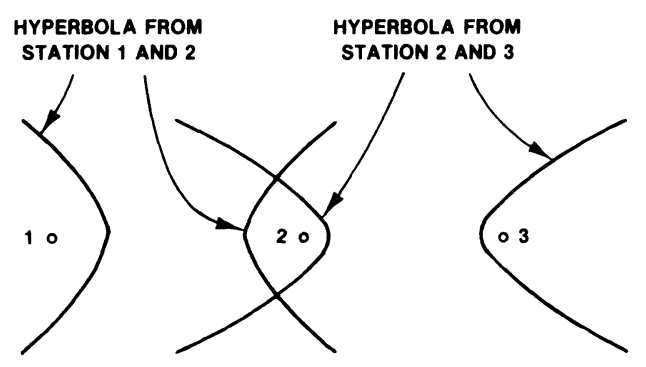

Fig. 1. Intersecting hyperbolas from three stations.

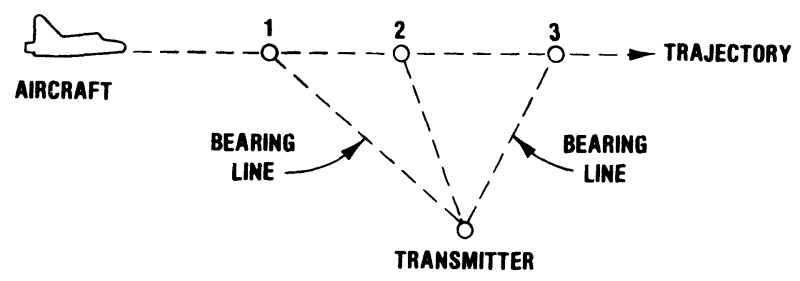

Fig. 2. Bearing lines from aircraft positions.

three dimensions and possibly other parameters such as the time of emission of the radiation. A set of $N$ measurements $r_{i}, i=1,2, \ldots, N$, is collected at various positions. In the absence of random measurement errors, $r_{i}$ is equal to a known function $f_{i}(\boldsymbol{x})$. In the presence of additive errors,

$r_{i}=f_{i}(\boldsymbol{x})+n_{i}, \quad i=1,2, \ldots, N$.

These $N$ equations can be written as a single equation for $N$-dimensional column vectors:

$r=f(x)+n$.

The measurement error $\boldsymbol{n}$ is assumed to be a multivariate random vector with an $N \times N$ positive-definite covariance matrix

$\boldsymbol{N}=E\left[(\boldsymbol{n}-E[\boldsymbol{n}])(\boldsymbol{n}-E[\boldsymbol{n}])^{\mathrm{T}}\right]$

where $E[\quad]$ denotes the expected value and the superscript $\mathrm{T}$ denotes the transpose.

If $\boldsymbol{x}$ is regarded as an unknown but nonrandom vector and $\boldsymbol{n}$ is assumed to have a zero mean and a Gaussian distribution, then the conditional density function of $r$ given $\boldsymbol{x}$ is

$$
\begin{aligned}
p(\boldsymbol{r} \mid \boldsymbol{x})= & \frac{1}{(2 \pi)^{N / 2}|\boldsymbol{N}|^{1 / 2}} \\
& \exp \left\{-(1 / 2)[\boldsymbol{r}-\boldsymbol{f}(\boldsymbol{x})]^{\mathrm{T}} \boldsymbol{N}^{-1}[\boldsymbol{r}-\boldsymbol{f}(\boldsymbol{x})]\right\}
\end{aligned}
$$

where $|N|$ denotes the determinant of $N$ and the superscript -1 denotes the inverse. Because $N$ is symmetric and positive definite, its inverse exists. The maximum likelihood estimator is that value of $\boldsymbol{x}$ which maximizes (4). Thus the maximum likelihood estimator minimizes the quadratic form

$Q(x)=[r-f(x)]^{\mathrm{T}} N^{-1}[r-f(x)]$.

Minimization of $Q(x)$ is a reasonable criterion for determination of an estimator even when the additive error cannot be assumed to be Gaussian. In this case, the resulting estimator is called the least squares estimator and $N^{-1}$ is regarded as a matrix of weighting coefficients.

In general, $f(x)$ is a nonlinear vector function. To determine a reasonably simple estimator, $\boldsymbol{f}(\boldsymbol{x})$ can be linearized by expanding it in a Taylor series about a reference point specified by the vector $x_{0}$ and retaining the first two terms; that is, we use

$f(x) \simeq f\left(x_{0}\right)+G\left(x-x_{0}\right)$

where $\boldsymbol{x}$ and $\boldsymbol{x}_{0}$ are $n \times 1$ column vectors and $\boldsymbol{G}$ is the $N$ $\times n$ matrix of derivatives evaluated at $x_{0}$ :

$$
\boldsymbol{G}=\left[\begin{array}{ccc}
\left.\frac{\partial f_{1}}{\partial x_{1}}\right|_{x=x_{0}} & \cdots & \left.\frac{\partial f_{1}}{\partial x_{n}}\right|_{x=x_{0}} \\
\vdots & \vdots \\
\left.\frac{\partial f_{N}}{\partial x_{1}}\right|_{x=x_{0}} & \cdots & \left.\frac{\partial f_{N}}{\partial x_{n}}\right|_{x=x_{0}}
\end{array}\right]
$$

Each row of this matrix is the gradient vector of one of the components of $\boldsymbol{f}(\boldsymbol{x})$. The vector $\boldsymbol{x}_{0}$ could be an estimate of $\boldsymbol{x}$ determined from a previous iteration of the estimation procedure or based upon a priori information. It is assumed in the subsequent analysis that $\boldsymbol{x}_{0}$ is sufficiently close to $x$ that (6) is an accurate approximation.

Combining (5) and (6) gives

$$
Q(x)=\left(r_{1}-G x\right)^{\mathrm{T}} N^{-1}\left(r_{1}-G x\right)
$$

where

$r_{1}=r-f\left(x_{0}\right)+G x_{0}$.

To determine the necessary condition for the estimator $\hat{\boldsymbol{x}}$ that minimizes $Q(\boldsymbol{x})$, we calculate the gradient of $Q(\boldsymbol{x})$, defined by

$\nabla_{x} Q(\boldsymbol{x})=\left[\frac{\partial Q}{\partial x_{1}} \frac{\partial Q}{\partial x_{2}} \cdots \frac{\partial Q}{\partial x_{n}}\right]^{\mathrm{T}}$

and then solve for the $x$ such that $\nabla_{x} Q(x)=0$. From its definition, $N$ is a symmetric matrix; that is, $N^{\mathrm{T}}=N$. Since $\left(N^{-1}\right)^{\mathrm{T}}=\left(\boldsymbol{N}^{\mathrm{T}}\right)^{-1}$, it follows that $\left(\boldsymbol{N}^{-1}\right)^{\mathrm{T}}=N^{-1}$, which implies that $N^{-1}$ is a symmetric matrix. Therefore,

$\left.\nabla_{x} Q(x)\right|_{x=\dot{x}}=2 G^{\mathrm{T}} N^{-1} \boldsymbol{G} \hat{\boldsymbol{x}}-2 \boldsymbol{G}^{\mathrm{T}} \boldsymbol{N}^{-1} \boldsymbol{r}_{1}=\mathbf{0}$.

We assume that the matrix $G^{\mathrm{T}} \boldsymbol{N}^{-1} \boldsymbol{G}$ is nonsingular. Thus the solution of (11) is

$$
\begin{aligned}
\hat{\boldsymbol{x}} & =\left(\boldsymbol{G}^{\mathrm{T}} \boldsymbol{N}^{-1} \boldsymbol{G}\right)^{-1} G^{\mathrm{T}} \boldsymbol{N}^{-1} \boldsymbol{r}_{1} \\
& =\boldsymbol{x}_{0}+\left(\boldsymbol{G}^{\mathrm{T}} \boldsymbol{N}^{-1} \boldsymbol{G}\right)^{-1} \boldsymbol{G}^{\mathrm{T}} \boldsymbol{N}^{-1}\left[\boldsymbol{r}-\boldsymbol{f}\left(\boldsymbol{x}_{0}\right)\right] .
\end{aligned}
$$

Using (12), direct calculation shows that (8) can be written in the form 


$$
\begin{aligned}
Q(x)=[ & x-\hat{x}]^{\mathrm{T}} G^{\mathrm{T}} N^{-1} G[\boldsymbol{x}-\hat{\boldsymbol{x}}] \\
& -\boldsymbol{r}_{1}^{\mathrm{T}} \boldsymbol{N}^{-1} \boldsymbol{G}\left(\boldsymbol{G}^{\mathrm{T}} \boldsymbol{N}^{-1} \boldsymbol{G}\right)^{-1} \boldsymbol{r}_{1}+\boldsymbol{r}_{1}^{\mathrm{T}} \boldsymbol{N}^{-1} \boldsymbol{r}_{1}
\end{aligned}
$$

where only the first term depends upon $\boldsymbol{x}$. Since $\boldsymbol{N}$ is symmetric and positive definite, it has positive eigenvalues. If $\mathrm{Ne}=\lambda \boldsymbol{e}$, then $\boldsymbol{N}^{-1} \boldsymbol{e}=\lambda^{-1} e$. Thus if $e$ is an eigenvector of $\boldsymbol{N}$ with eigenvalue $\lambda$, then $\boldsymbol{e}$ is also an eigenvector of $N^{-1}$ with eigenvalue $1 / \lambda$. Since it is symmetric and its eigenvalues are positive, $N^{-1}$ is positive definite. Therefore, $\boldsymbol{x}=\hat{\boldsymbol{x}}$ minimizes $Q(\boldsymbol{x})$. The estimator of (12) is called the linearized least squares estimator.

Substituting (2) into (12) and rearranging terms, the expression for $\hat{\boldsymbol{x}}$ can be written in the form

$$
\begin{aligned}
\hat{\boldsymbol{x}}=\boldsymbol{x}+\left(G^{\mathrm{T}} N^{-1} G\right)^{-1} G^{\mathrm{T}} N^{-1}[ & f(x)-f\left(x_{0}\right) \\
& \left.-G\left(x-x_{0}\right)+n\right]
\end{aligned}
$$

which shows how the estimator error is affected by the linearization error and the noise. The bias of the estimator $\hat{\boldsymbol{x}}$ is defined as $\boldsymbol{b}=E[\hat{\boldsymbol{x}}]-\boldsymbol{x}$. Using (14), we obtain

$$
\begin{aligned}
b=\left(G^{\mathrm{T}} \boldsymbol{N}^{-1} G\right)^{-1} G^{\mathrm{T}} \boldsymbol{N}^{-1} & \left\{\boldsymbol{f}(\boldsymbol{x})-\boldsymbol{f}\left(\boldsymbol{x}_{0}\right)\right. \\
& \left.-\boldsymbol{G}\left(\boldsymbol{x}-\boldsymbol{x}_{0}\right)+E[\boldsymbol{n}]\right\} .
\end{aligned}
$$

If $\boldsymbol{f}(\boldsymbol{x})$ is linear, as in (6), and $E[\boldsymbol{n}]=\mathbf{0}$, then the least squares estimator is unbiased. If systematic errors occur in the measurements, then $E[\boldsymbol{n}] \neq \mathbf{0}$. To minimize the estimator bias due to systematic errors, the magnitude of each $E\left[n_{i}\right]$ should be minimized through system calibrations. If some of the $E\left[n_{i}\right]$ are known functions of various parameters and $N$ is sufficiently large, these parameters can be made components of the vector $\boldsymbol{x}$ and estimated along with the other components of $\boldsymbol{x}$. The bias due to the nonlinearity of $f(x)$ can be estimated by expanding $f(x)$ in a Taylor series about $x_{0}$ and retaining second-order terms.

Let $\boldsymbol{P}$ denote the covariance matrix of $\hat{\boldsymbol{x}}$. Equation (14) yields

$\boldsymbol{P}=E\left[(\hat{\boldsymbol{x}}-E[\hat{\boldsymbol{x}}])(\hat{\boldsymbol{x}}-E[\hat{\boldsymbol{x}}])^{\mathrm{T}}\right]=\left(\boldsymbol{G}^{\mathrm{T}} \boldsymbol{N}^{-1} \boldsymbol{G}\right)^{-1}$.

The diagonal elements of $\boldsymbol{P}$ give the variances of the errors in the estimated components of $\boldsymbol{x}$. Since $\boldsymbol{P}$ is part of the estimator given by (12), one can compute both estimate and covariance simultaneously. If $\boldsymbol{n}$ is zero-mean Gaussian, the maximum likelihood or least squares estimator for the linearized model is the same as the minimum variance unbiased estimator [3].

The measurement error vector $\boldsymbol{n}$ is assumed to encompass all the contributions to error, including uncertainties in the system or physical parameters, such as the station coordinates or the speed of propagation. If $q$ is a vector of the parameters, then the measurement vector $\boldsymbol{r}$ can often be expressed as

$r=f_{1}(x, q)+n_{1}$

where $f_{1}(\quad)$ is a vector function and $n_{1}$ is the random error due to causes unrelated to uncertainties in $\boldsymbol{q}$. Let $\boldsymbol{q}_{0}$ denote the assumed value of $\boldsymbol{q}$. If $\boldsymbol{q}_{0}$ is sufficiently close to $\boldsymbol{q}$, then a Taylor series expansion yields

$f_{1}(x, q) \simeq f_{1}\left(x, q_{0}\right)+G_{1}\left(q-q_{0}\right)$

where $G_{1}$ is the matrix of derivatives with respect to $q$ evaluated at $\boldsymbol{q}_{0}$. Equation (2) results from making the identifications

$f(x)=f_{1}\left(x, q_{0}\right), \quad n=G_{1}\left(q-q_{0}\right)+n_{1}$.

If $\boldsymbol{q}$ is nonrandom, then the parameter uncertainties ultimately contribute to the bias of the least squares estimator. If $\boldsymbol{q}$ is random, then the variance and possibly the bias are affected.

Any a priori information can be incorporated into the estimation procedure in several ways. It can be used to select an accurate reference point $\boldsymbol{x}_{0}$ for the first iteration of the least squares estimator. If the transmitter is known to be located within a region, but the estimated position is outside this region, a logical procedure is to change the estimate to the point in the region that is closest to the original estimate. If an a priori distribution function for the transmitter position can be specified, a Bayesian estimator can be determined. However, the Bayesian estimator is usually too complex a mathematical function to yield a simple computational algorithm unless simplifying assumptions are made about the a priori distribution [4].

The location estimate can be continually refined if a sequence of measurements is taken. If successive measurements are uncorrelated, a new least squares estimate can be determined by combining new measurements with the old estimate [3]. Since measurements do not have to be stored after processing, a significant computational savings is sometimes possible.

\section{ESTIMATOR ACCURACY}

If $\boldsymbol{r}$ is a Gaussian random vector, then (12) indicates that $\hat{\boldsymbol{x}}$ is a Gaussian random vector. Its probability density function is

$$
\begin{aligned}
\boldsymbol{f}_{\hat{\boldsymbol{x}}}(\boldsymbol{\xi})=\left[(2 \pi)^{n / 2}|\boldsymbol{P}|^{1 / 2}\right]^{-1} & \\
& \exp \left[-(1 / 2)(\boldsymbol{\xi}-\boldsymbol{m})^{\mathrm{T}} \boldsymbol{P}^{-1}(\boldsymbol{\xi}-\boldsymbol{m})\right]
\end{aligned}
$$

where $\boldsymbol{m}=E[\hat{\boldsymbol{x}}]$ is the mean vector, and

$\boldsymbol{P}=E\left[(\hat{\boldsymbol{x}}-\boldsymbol{m})(\hat{\boldsymbol{x}}-\boldsymbol{m})^{\mathrm{T}}\right]$

is the covariance matrix given by (16). By definition, $P$ is symmetric and positive semidefinite. Thus it has nonnegative eigenvalues. Equation (16) indicates that $\boldsymbol{P}^{-1}$ exists and in equal to $\boldsymbol{G}^{\mathrm{T}} \boldsymbol{N}^{-1} \boldsymbol{G}$. Therefore, $\boldsymbol{P}$ does not have zero as an eigenvalue. Thus $\boldsymbol{P}$ is positive definite.

The loci of constant density function values are described by equations of the form

$(\boldsymbol{\xi}-\boldsymbol{m})^{\mathrm{T}} \boldsymbol{P}^{-1}(\boldsymbol{\xi}-\boldsymbol{m})=\kappa$

where $\kappa$ is a constant that determines the size of the $n$ dimensional region enclosed by the surface. In two 
dimensions, the surface is an ellipse; in three dimensions, it is an ellipsoid; in the general case of $n$ dimensions, it may be considered a hyperellipsoid. Unless $\boldsymbol{P}$ is a diagonal matrix, the principal axes of the hyperellipsoids are not aligned with the coordinate axes.

The probability that $\hat{\boldsymbol{x}}$ lies inside the hyperellipsoid of (22) is

$P_{e}(\kappa)=\iint_{R} \cdots \int \boldsymbol{f}_{\hat{x}}(\boldsymbol{\xi}) d \xi_{1} d \xi_{2} \cdots d \xi_{n}$

where the region of integration is

$R=\left\{\boldsymbol{\xi}:(\boldsymbol{\xi}-\boldsymbol{m})^{\mathrm{T}} \boldsymbol{P}^{-1}(\boldsymbol{\xi}-\boldsymbol{m}) \leq \boldsymbol{\kappa}\right\}$.

To reduce (23) to a single integral, we perform a succession of coordinate transformations. First, we translate the coordinate system so that its origin coincides with $\boldsymbol{m}$ by making the change of variables $\boldsymbol{\gamma}=\boldsymbol{\xi}-\boldsymbol{m}$. Since the Jacobian is unity, we obtain

$$
\begin{array}{r}
P_{e}(\kappa)=a \iint_{R_{1}} \cdots \int \exp \left(-\frac{1}{2} \boldsymbol{\gamma}^{\mathrm{T}} \boldsymbol{P}^{-1} \boldsymbol{\gamma}\right) \\
d \gamma_{1} d \gamma_{2} \cdots d \gamma_{n}
\end{array}
$$

where

$$
\begin{aligned}
R_{1} & =\left\{\boldsymbol{\gamma}: \boldsymbol{\gamma}^{\mathrm{T}} \boldsymbol{P}^{-1} \boldsymbol{\gamma} \leq \mathrm{\kappa}\right\} \\
a & =\left[(2 \pi)^{n / 2}|\boldsymbol{P}|^{1 / 2}\right]^{-1} .
\end{aligned}
$$

To simplify (25), we rotate the coordinate axes so that they are aligned with the principal axes of the hyperellipsoid. Because $\boldsymbol{P}$ is a symmetric positive-definite matrix, so is $\boldsymbol{P}^{-1}$. Therefore, an orthogonal matrix $\boldsymbol{A}$ (with eigenvectors as columns) exists that diagonalizes $\boldsymbol{P}^{-1}$. Thus $\boldsymbol{A}^{\mathrm{T}}=\boldsymbol{A}$ and

$$
\boldsymbol{A}^{\mathrm{T}} \boldsymbol{P}^{-1} \boldsymbol{A}=\left[\begin{array}{cccc}
\lambda_{1}^{-1} & & & 0 \\
& \lambda_{2}^{-1} & \ddots & \\
0 & & & \lambda_{n}^{-1}
\end{array}\right]=\left[\lambda^{-1}\right]
$$

where $\lambda_{1}, \lambda_{2}, \ldots, \lambda_{n}$ are the eigenvalues of $\boldsymbol{P}$. A rotation of axes results in new variables defined by

$\boldsymbol{\zeta}=\boldsymbol{A}^{\mathrm{T}} \boldsymbol{\gamma}$.

Since $\boldsymbol{A}^{\mathrm{T}} \boldsymbol{A}=\boldsymbol{I}$ and the determinant of the product of matrices is equal to the product of the determinants of the matrices, the determinant of $\boldsymbol{A}^{\mathrm{T}}$, which is the Jacobian of the transformation, is unity. Substituting (28) and (29) into (25) and (26) yields

$$
\begin{aligned}
P_{e}(\kappa) & =a \iint_{R_{2}} \cdots \int \exp \left(-\frac{1}{2} \zeta^{\mathrm{T}}\left[\lambda^{-1}\right] \zeta\right) d \zeta_{1} d \zeta_{2} \cdots d \zeta_{n} \\
& =a \iint_{R_{2}} \cdots \int \exp \left(-\frac{1}{2} \sum_{i=1}^{n} \frac{\zeta_{i}^{2}}{\lambda_{i}}\right) d \zeta_{1} d \zeta_{2} \cdots d \zeta_{n}
\end{aligned}
$$

where
$R_{2}=\left\{\xi: \sum_{i=1}^{n} \frac{\zeta_{i}^{2}}{\lambda_{i}} \leq \kappa\right\}$,

and the $\zeta_{i}$ are the components of $\zeta$. Regions $R_{2}$ is the interior of a hyperellipsoid with principal axes of lengths $2 \sqrt{\kappa \lambda_{i}}, i=1,2, \ldots, n$. By introducing new variables

$\eta_{i}=\zeta_{i} / \sqrt{\lambda_{i}}, \quad i=1,2, \ldots, n$

we can simplify (30) further. Since the determinant of $\boldsymbol{P}$ is equal to the product of the eigenvalues of $\boldsymbol{P},(27)$ and (30) to (32) give

$$
\begin{aligned}
& P_{e}(\kappa)=(2 \pi)^{-n / 2} \iint \\
& \sum_{i=1}^{n} \eta_{i}^{2} \leq \kappa \\
& \exp \left(-\frac{1}{2} \sum_{i=1}^{n} \eta_{i}^{2}\right) d \eta_{1} d \eta_{2} \cdots d \eta_{n} .
\end{aligned}
$$

The region of integration, which is indicated below the integral signs, is the interior of a hypersphere.

It is shown below that the volume of an $n$ dimensional hypersphere of radius

$\rho=\left(\sum_{i=1}^{n} \eta_{i}^{2}\right)^{1 / 2}$

is

$V_{n}(\rho)=\frac{\pi^{n / 2} \rho^{n}}{\Gamma(n / 2+1)}$

where $\Gamma(\quad)$ is the gamma function. Therefore, the differential volume between $\rho$ and $\rho+d \rho$ is

$d v=\frac{n \pi^{n / 2} \rho^{n-1}}{\Gamma(n / 2+1)} d \rho$

and (33) can be reduced to

$P_{e}(\kappa)=\frac{n}{2^{n / 2} \Gamma(n / 2+1)} \int_{0}^{\sqrt{\kappa}} \rho^{n-1} \exp \left(-\frac{\rho^{2}}{2}\right) d \rho$.

For $n=1,2$, and 3, this integral can be expressed in simpler terms:

$$
\begin{aligned}
P_{e}(\kappa)= & \operatorname{erf}(\sqrt{\kappa} / 2), \quad n=1 \\
P_{e}(\kappa)= & 1-\exp (-\kappa / 2), \quad n=2 \\
P_{e}(\kappa)= & \operatorname{erf}(\sqrt{\kappa} / 2) \\
& -\sqrt{2 \kappa} / \pi \exp (-\kappa / 2), \quad n=3
\end{aligned}
$$

where the error function is defined by

$\operatorname{erf}(x)=\frac{2}{\sqrt{\pi}} \int_{0}^{x} \exp \left(-t^{2}\right) d t$

Equation (40) is obtained by integration by parts.

To verify (35), we define the volume of a hypersphere of radius $\rho$ by 
$V_{n}(\rho)=\iint_{\sum_{i=1}^{n} x_{i}^{2} \leq \rho^{2}} \cdots \int d x_{1} d x_{2} \cdots d x_{n}$.

A change of coordinates shows that

$V_{n}(\rho)=\rho^{n} V_{n}(1)$

where $V_{n}(1)$ is the volume of a unit hypersphere.

Straightforward calculations give

$V_{1}(1)=2, \quad V_{2}(1)=\pi$

where the "volumes" are a length and an area, respectively. We define the sets

$B=\left\{\left(x_{1}, x_{2}\right): x_{1}^{2}+x_{2}^{2} \leq 1\right\}$

$C=\left\{\left(x_{3}, \ldots, x_{n}\right): \sum_{i=3}^{n} x_{i}^{2} \leq 1-x_{1}^{2}-x_{2}^{2}\right\}$.

For $n \geq 3$, Fubini's theorem for interchanging the order of integrations [5] and a change of coordinates in (42) give

$$
\begin{aligned}
V_{n}(1) & =\iint_{B} d x_{1} d x_{2}, \iint_{C} \ldots \int d x_{3} \cdots d x_{n} \\
& =\iint_{B} V_{n-2}\left(\sqrt{1-x_{1}^{2}-x_{2}^{2}}\right) d x_{1} d x_{2} .
\end{aligned}
$$

Equation (43) and further coordinate changes yield

$$
\begin{aligned}
V_{n}(1) & =V_{n-2}(1) \iint_{B}\left(1-x_{1}^{2}-x_{2}^{2}\right)^{(n-2) / 2} d x_{1} d x_{2} \\
& =V_{n-2}(1) \int_{0}^{2 \pi} \int_{0}^{1}\left(1-r^{2}\right)^{(n-2) / 2} r d r d \theta \\
& =\pi V_{n-2}(1) \int_{0}^{1} x^{(n-2) / 2} d x \\
& =2 \pi V_{n-2}(1) / n .
\end{aligned}
$$

By induction, this recursion relation and (44) imply that

$$
\begin{aligned}
V_{2 m}(1) & =\frac{(2 \pi)^{m}}{2 \cdot 4 \cdots(2 m)} ; \\
V_{2 m-1}(1) & =\frac{2(2 \pi)^{m-1}}{1 \cdot 3 \cdots(2 m-1)}, \quad m=1,2, \ldots .
\end{aligned}
$$

We can express $V_{n}(1)$ in terms of a compact formula by using the properties of the gamma function: $\Gamma(t+1)=$ $t \Gamma(t) ; \Gamma(1)=1 ; \Gamma(1 / 2)=\sqrt{\pi}$. We obtain

$V_{n}(1)=\frac{\pi^{n / 2}}{\Gamma(n / 2+1)}, \quad n=1,2, \ldots$.

Combining (43) and (50) yields (35).

If $P_{e}$ is specified, say $P_{e}=1 / 2$, then (37), (38), (39), or (40) can be solved numerically to determine the corresponding value of $\kappa$, which in turn defines a hyperellipsoid by (22). The concentration ellipsoid corresponding to probability $P_{e}$ is defined to be the particular hyperellipsoid for which $P_{e}$ is the probability that $\hat{\boldsymbol{x}}$ lies inside it. Thus the concentration ellipsoid is a multidimensional measure of accuracy for an unbiased estimator.

A scalar measure of estimator accuracy is the rootmean-square error $\epsilon_{r}$, which is defined by

$\epsilon_{r}^{2}=E\left[\sum_{i=1}^{n}\left(\hat{x}_{i}-x_{i}\right)^{2}\right]$.

Expanding (51) and using (21), we obtain

$\boldsymbol{\epsilon}_{r}^{2}=\operatorname{tr}(\boldsymbol{P})+\sum_{i=1}^{n} b_{i}^{2}$

where $\operatorname{tr}(\boldsymbol{P})$ denotes the trace of $\boldsymbol{P}$ and $b_{i}=E\left[\hat{x}_{i}\right]-x_{i}$ denotes a component of the bias vector $\boldsymbol{b}$.

\section{TWO-DIMENSIONAL ESTIMATORS}

For the estimator of a two-dimensional vector, such as position coordinates on the surface of the Earth, the bivariate covariance matrix can be expressed as

$\boldsymbol{P}=\left[\begin{array}{cc}\sigma_{1}^{2} & \sigma_{12} \\ \sigma_{12} & \sigma_{2}^{2}\end{array}\right]$.

A straightforward calculation yields the eigenvalues:

$\lambda_{1}=\frac{1}{2}\left[\sigma_{1}^{2}+\sigma_{2}^{2}+\sqrt{\left(\sigma_{1}^{2}-\sigma_{2}^{2}\right)^{2}+4 \sigma_{12}^{2}}\right]$

$\lambda_{2}=\frac{1}{2}\left[\sigma_{1}^{2}+\sigma_{2}^{2}-\sqrt{\left(\sigma_{1}^{2}-\sigma_{2}^{2}\right)^{2}+4 \sigma_{12}^{2}}\right]$

where the positive square root is used. By definition, $\lambda_{1}$ $\geq \lambda_{2}$.

Suppose that new coordinates are defined by rotating the axes of the old coordinate system counterclockwise through an angle $\theta$, as shown in Fig. 3. A vector

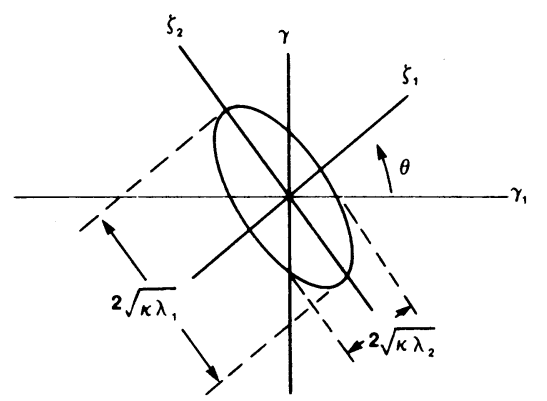

Fig. 3. Concentration ellipse and coordinate axes.

represented by $\boldsymbol{\gamma}$ in the old coordinates is represented in the new coordinates by $\zeta=A^{\mathrm{T}} \boldsymbol{\gamma}$, where $\boldsymbol{A}$ is the orthogonal matrix

$\boldsymbol{A}=\left[\begin{array}{rr}\cos \theta & -\sin \theta \\ \sin \theta & \cos \theta\end{array}\right]$. 
From (53) and (56), direct but lengthy calculation shows that $\boldsymbol{A}^{\mathrm{T}} \boldsymbol{P}^{-1} \boldsymbol{A}$ is a diagonal matrix and the columns of $\boldsymbol{A}$ are eigenvectors if

$\theta=\frac{1}{2} \tan ^{-1}\left(\frac{2 \sigma_{12}}{\sigma_{1}^{2}-\sigma_{2}^{2}}\right), \quad-\frac{\pi}{4} \leq \theta \leq \frac{\pi}{4}$.

If $\sigma_{1}^{2}=\sigma_{2}^{2}$ and $\sigma_{12}=0$, we take $\theta=0$. Since the determinant of a matrix is equal to the product of the eigenvalues, $\lambda_{1} \lambda_{2}=\sigma_{1}^{2} \sigma_{2}^{2}-\sigma_{12}^{2}$. Using this result, the diagonal matrix can be written in the form

$$
\begin{array}{ll}
{\left[\lambda^{-1}\right]=\left[\begin{array}{ll}
\lambda_{1}^{-1} & 0 \\
0 & \lambda_{2}^{-1}
\end{array}\right], \quad \sigma_{1}^{2} \geq \sigma_{2}^{2}} \\
{\left[\lambda^{-1}\right]=\left[\begin{array}{ll}
\lambda_{2}^{-1} & 0 \\
0 & \lambda_{1}^{-1}
\end{array}\right], \quad \sigma_{1}^{2}<\sigma_{2}^{2} .}
\end{array}
$$

Since $\boldsymbol{P}^{-1}$ exists according to (16), neither eigenvalue can equal zero and $\left[\lambda^{-1}\right]$ is well defined.

A concentration ellipse defined by $\boldsymbol{\gamma}^{\mathrm{T}} \boldsymbol{P}^{-1} \boldsymbol{\gamma}=\kappa$ in the old coordinates is described by $\left(\zeta_{1} / \lambda_{1}\right)^{2}+\left(\zeta_{2} / \lambda_{2}\right)=$ $\kappa$ or by $\left(\zeta_{1} / \lambda_{2}\right)^{2}+\left(\zeta_{2} / \lambda_{1}\right)^{2}=\kappa$ in the new coordinates, a fact which indicates that the new axes coincide with the principal axes of the ellipse. Thus (57) represents the angular offset of one of the principal axes of the ellipse relative to the old coordinate axes. Fig. 3 depicts a concentration ellipse and the appropriate angle of axis rotation. Since $\lambda_{1} \geq \lambda_{2}$, the major and minor axes have lengths $2 \sqrt{\kappa \lambda_{1}}$ and $2 \sqrt{\kappa \lambda_{2}}$, respectively. If the ellipse encloses a region that includes a Gaussian random vector with probability $P_{e}$, then (39) implies that

$\kappa=-2 \ln \left(1-P_{e}\right)$.

Suppose that a two-dimensional Gaussian random vector describes the estimated location of a transmitter. A crude but simple measure of accuracy is the circular error probable (CEP). The CEP is defined as the radius of the circle that has its center at the mean and contains half the realizations of the random vector. The CEP is a measure of the uncertainty in the location estimator $\hat{\boldsymbol{x}}$ relative to its mean $E[\hat{x}]$. If the location estimator is unbiased, the CEP is a measure of the estimator uncertainty relative to the true transmitter position. If the magnitude of the bias vector is bounded by $B$, then with a probability of onehalf, a particular estimate is within a distance of $B+$ CEP from the true position. The geometrical relations are depicted in Fig. 4.

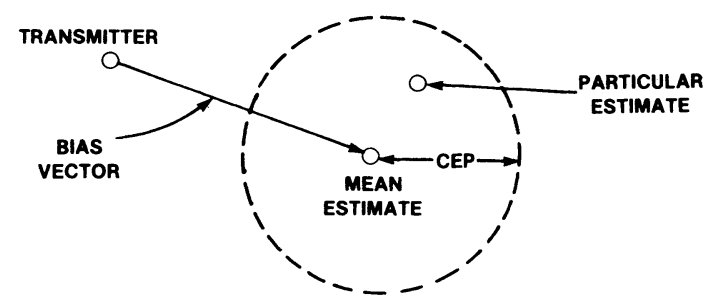

Fig. 4. Geometry of transmitter position, mean location estimate, CEP, estimator bias vector, and particular location estimate.
From the definition, it follows that we can determine the CEP by solving the equation

$\frac{1}{2}=\iint_{R} f_{\hat{x}}(\boldsymbol{\xi}) d \xi_{1} d \xi_{2}$

where

$R=\{\boldsymbol{\xi}:|\boldsymbol{\xi}-\boldsymbol{m}| \leq \mathrm{CEP}\}$.

In a manner analogous to the derivation of (30), we successively translate and rotate coordinates to obtain

$\frac{1}{2}=\frac{1}{2 \pi \sqrt{\lambda_{1} \lambda_{2}}} \iint_{R_{1}} \exp \left(-\frac{1}{2} \sum_{i=1}^{2} \frac{\zeta_{i}^{2}}{\lambda_{i}}\right) d \zeta_{1} d \zeta_{2}$

where

$R_{1}=\left\{\left(\zeta_{1}, \zeta_{2}\right):\left(\zeta_{1}^{2}+\zeta_{2}^{2}\right)^{1 / 2} \leq \mathrm{CEP}\right\}$

and the $\lambda_{i}$ are given by (54) and (55). Changing to polar coordinates by substituting $\zeta_{1}=r \cos \theta$ and $\zeta_{2}=r \sin$ $\theta$, we get

$$
\begin{aligned}
\pi \sqrt{\lambda_{1} \lambda_{2}}= & \int_{0}^{2 \pi} \int_{0}^{\mathrm{CEP}} r \\
& \exp \left[-\frac{r^{2}}{2}\left(\frac{\cos ^{2} \theta}{\lambda_{1}}+\frac{\sin ^{2} \theta}{\lambda_{2}}\right)\right] d r d \theta .
\end{aligned}
$$

To simplify (65), we do some preliminary manipulations. The modified Bessel function of the first kind and zero order can be expressed as

$I_{0}(x)=\frac{1}{2 \pi} \int_{0}^{2 \pi} \exp (x \cos \theta) d \theta$.

Because of the periodicity of the integrand, we also have

$I_{0}(x)=\frac{1}{2 \pi} \int_{2 \pi n}^{2 \pi(n+1)} \exp (x \cos \theta) d \theta$

for any integer $n$. Adding $m$ equations of this form with successive values of $n$, we obtain

$m I_{0}(x)=\frac{1}{2 \pi} \int_{0}^{2 \pi m} \exp (x \cos \theta) d \theta, \quad m=1,2, \ldots$.

Changing coordinates with $\theta=m \phi$ gives

$I_{0}(x)=\frac{1}{2 \pi} \int_{0}^{2 \pi} \exp (x \cos m \phi) d \phi, \quad m=1,2, \ldots$.

Trigonometric identities yield

$$
\begin{aligned}
\frac{\cos ^{2} \theta}{\lambda_{1}}+\frac{\sin ^{2} \theta}{\lambda_{2}}=\frac{1}{2 \lambda_{1}} & +\frac{1}{2 \lambda_{2}} \\
& +\left(\frac{1}{2 \lambda_{1}}-\frac{1}{2 \lambda_{2}}\right) \cos 2 \theta .
\end{aligned}
$$

Substituting (70) into (65) and using (69), we obtain 


$$
\begin{aligned}
\frac{\sqrt{\lambda_{1} \lambda_{2}}}{2}=\int_{0}^{\mathrm{CEP}} r \exp [- & \left.\left(\frac{1}{4 \lambda_{1}}+\frac{1}{4 \lambda_{2}}\right) r^{2}\right] \\
& I_{0}\left[\left(\frac{1}{4 \lambda_{2}}-\frac{1}{4 \lambda_{1}}\right) r^{2}\right] d r .
\end{aligned}
$$

A final change of coordinates yields

$$
\begin{aligned}
& \frac{1}{4 \gamma^{2}}\left(1+\gamma^{2}\right)=\int_{0}^{\left[(\mathrm{CEP})^{2} / 4 \lambda_{2}\right]\left(1+\gamma^{2}\right)} \exp (-x) \\
& I_{0}\left(\frac{1-\gamma^{2}}{1+\gamma^{2}} x\right) d x, \quad \gamma^{2}=\frac{\lambda_{2}}{\lambda_{1}} .
\end{aligned}
$$

The form of this relation implies that the CEP has the form CEP $=\sqrt{\lambda_{2}} f(\gamma)$ for some function $f(\quad)$. If $\sigma_{12}=$ 0 and $\sigma_{1}=\sigma_{2}=\sigma$, then $\lambda_{1}=\lambda_{2}=\sigma^{2}$ and (72) can be solved to show that CEP $=1.177 \sigma$. In the general case where $\lambda_{1} \neq \lambda_{2}$, numerical integration is necessary to solve for the CEP. A simple approximation that is consistent with the preceding observations is

$\mathrm{CEP} \approx 0.563 \sqrt{\lambda_{1}}+0.614 \sqrt{\lambda_{2}}$

which is accurate to within 1 percent for $\gamma \simeq 0.3$ or larger, underestimates the CEP by less than 10 percent for $0.1<\gamma<0.3$, and underestimates by less than 20 percent elsewhere. Although approximations that are more accurate for small $\gamma$ are easily produced, they are usually irrelevant because the eccentricity of the concentration ellipse for small $\gamma$ may be too pronounced for the CEP to be an adequate performance measure. An approximation that is accurate to within approximately 10 percent for all values of $\gamma$ is

$\mathrm{CEP} \approx 0.75 \sqrt{\lambda_{1}+\lambda_{2}}=0.75 \sqrt{\sigma_{1}^{2}+\sigma_{2}^{2}}$

where the last relation follows from the fact that the trace of a matrix is equal to the sum of its eigenvalues. Above $\gamma \approx 0.4$, this approximation underestimates the CEP; below $\gamma \approx 0.4$, it overestimates the CEP. For an unbiased estimator, (52) implies that $\mathrm{CEP} \approx 0.75 \epsilon_{r}$.

\section{HYPERBOLIC LOCATION SYSTEMS}

Suppose that the arrival times $t_{1}, t_{2}, \ldots, t_{N}$ of a signal transmitted at time $t_{0}$ are measured at $N$ stations having positions specified by the column vectors $s_{1}, s_{2}, \ldots, s_{N}$. The geometrical configuration is illustrated in Fig. 5. If

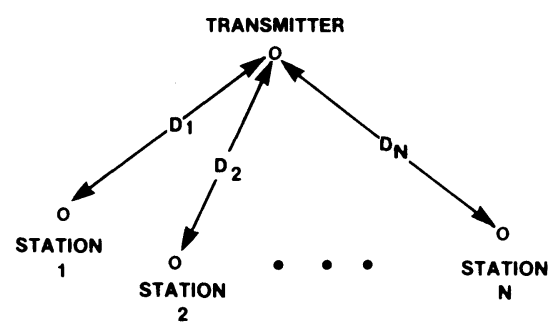

Fig. 5. Geometry of transmitter and $N$ stations. the signal velocity is $c$ and if $D_{i}$ is the propagation path length between the transmitter and station $i$, then

$t_{i}=t_{0}+D_{i} / c+\epsilon_{i}, \quad i=1,2, \ldots, N$.

The arrival-time measurement error $\epsilon_{i}$ accounts for propagation anomalies, receiver noise, and errors in the assumed station positions. In matrix form, (75) becomes

$\boldsymbol{t}=t_{0} \mathbf{1}+\boldsymbol{D} / c+\boldsymbol{\epsilon}$

where $\boldsymbol{t}, \boldsymbol{D}$, and $\boldsymbol{\epsilon}$ are $N$-dimensional column vectors with components $t_{i}, D_{i}$, and $\epsilon_{i}, i=1,2, \ldots, N$, respectively, and 1 is a column vector of ones.

Suppose that we seek to estimate both $t_{0}$ and the column vector $\boldsymbol{R}$, with components $x, y$, and $z$, that specifies the transmitter position. Equation (76) has the form of (2) with $\boldsymbol{r}=\boldsymbol{t}, \boldsymbol{f}(\boldsymbol{x})=t_{0} \mathbf{1}+\boldsymbol{D} / \boldsymbol{c}, \boldsymbol{n}=\boldsymbol{\epsilon}$, and $\boldsymbol{x}=\left[\begin{array}{llll}t_{0} & x & y & z\end{array}\right]^{\mathrm{T}}$. For line-of-sight propagation from the transmitter to the stations, $D_{i}=\left\|\boldsymbol{R}-s_{i}\right\|$, where \|\| represents the Euclidean norm. Let the column vector $\boldsymbol{R}_{0}$, with components $x_{0}, y_{0}$, and $z_{0}$, specify a reference point near the transmitter position. Let $D_{0 i}=\left\|\boldsymbol{R}_{0}-\boldsymbol{s}_{i}\right\|$ denote the distance from station $i$ to the reference point. Using (7) with $\boldsymbol{x}_{0}=\left[\begin{array}{llll}0 & x_{0} & y_{0} & z_{0}\end{array}\right]^{\mathrm{T}}$ after expressing each $\left\|\boldsymbol{R}-\boldsymbol{s}_{i}\right\|$ in terms of its components, we obtain

$\boldsymbol{G}=\left[\begin{array}{ll}1 & F / c\end{array}\right]$

where

$\boldsymbol{F}=\left[\begin{array}{c}\left(\boldsymbol{R}_{0}-s_{1}\right)^{\mathrm{T}} / D_{01} \\ \vdots \\ \left(\boldsymbol{R}_{0}-\boldsymbol{s}_{N}\right)^{\mathrm{T}} / D_{0 N}\end{array}\right]$.

Each row of $\boldsymbol{F}$ is the unit vector pointing from one of the stations to the reference point. Equation (12) with the above relations and substitutions gives the least squares or maximum likelihood estimator; (16) provides the covariance matrix of the estimator.

In hyperbolic systems, no attempt is made to estimate $t_{0}$. We eliminate it from consideration by measuring the relative arrival times:

$$
\begin{aligned}
t_{i}-t_{i+1}=\left(D_{i}-D_{i+1}\right) / c+ & n_{i}, \\
i & =1,2, \ldots, N-1
\end{aligned}
$$

where $n_{i}$ is the measurement error. Measuring time differences is not the only way to eliminate $t_{0}$, but it is the simplest. If the relative arrival times are determined by subtracting measured arrival times, then

$n_{i}=\epsilon_{i}-\epsilon_{i+1}, \quad i=1,2, \ldots, N-1$.

The $n_{i}$ have zero means if successive $\epsilon_{i}$ have equal means, even if the latter means are nonzero. A nonzero $E\left[n_{i}\right]$ may result from uncalibrated different time delays or unsynchronized clocks in two receivers. If the relative arrival times are determined by cross correlation, then (80) is not necessarily valid.

If the transmitter produces a sequence of pulses, the corresponding received pulses at stations $i$ and $i+1$ must be correctly associated in measuring the time difference $t_{i}-t_{i+1}$. A potential ambiguity arises when 
the time difference exceeds the time between successive pulse transmissions. This ambiguity may be resolved by using bearing measurements or a priori information to eliminate associations that lead to impossible location estimates.

In matrix form, (79) becomes

$\boldsymbol{H} \boldsymbol{t}=\boldsymbol{H D} / \boldsymbol{c}+\boldsymbol{n}$

where we use the $(N-1) \times N$ matrix

$\boldsymbol{H}=\left[\begin{array}{rrrrrr}1 & -1 & 0 & \cdots & 0 & 0 \\ 0 & 1 & -1 & \cdots & 0 & 0 \\ \vdots & \vdots & \vdots & & \vdots & \vdots \\ 0 & 0 & 0 & \cdots & 1 & -1\end{array}\right]$.

If $(80)$ is valid, then

$\boldsymbol{n}=\boldsymbol{H \epsilon}$.

Since we seek to estimate the position vector $R,(81)$ has the form of (2) with $\boldsymbol{r}=\boldsymbol{H} \boldsymbol{t}, \boldsymbol{f}(\boldsymbol{x})=\boldsymbol{H D} / \boldsymbol{c}$, and $\boldsymbol{x}$ $=\boldsymbol{R}$. A direct calculation of $\boldsymbol{G}$ yields

$\boldsymbol{G}=\boldsymbol{H F} / \boldsymbol{c}$

where $\boldsymbol{F}$ is defined by (78). Let $N_{\epsilon}$ denote the covariance matrix of the arrival-time errors. If (83) holds, then the covariance matrix of the measurement errors, defined by (3), is related to $N_{\epsilon}$ by

$\boldsymbol{N}=\boldsymbol{H} \boldsymbol{N}_{\epsilon} \boldsymbol{H}^{\mathrm{T}}$.

Using (84), equation (12) implies that the least squares estimator is

$\hat{\boldsymbol{R}}=\boldsymbol{R}_{0}+c\left(\boldsymbol{F}^{\mathrm{T}} \boldsymbol{H}^{\mathrm{T}} \boldsymbol{N}^{-1} \boldsymbol{H} \boldsymbol{F}\right)^{-1} \boldsymbol{F}^{\mathrm{T}} \boldsymbol{H}^{\mathrm{T}} \boldsymbol{N}^{-1}\left(\boldsymbol{H} \boldsymbol{t}-\boldsymbol{H} \boldsymbol{D}_{0} / c\right)$

where $D_{0}$ has components $D_{0 i}, i=1,2, \ldots, N$. The estimator is unbiased if $\boldsymbol{n}$ is a zero-mean random variable and the linearization error is negligible. The covariance matrix of $\hat{\boldsymbol{R}}$, given by (16), is

$\boldsymbol{P}=c^{2}\left(\boldsymbol{F}^{\mathrm{T}} \boldsymbol{H}^{\mathrm{T}} \boldsymbol{N}^{-1} \boldsymbol{H} \boldsymbol{F}\right)^{-1}$.

Equation (86) is valid for line-of-sight propagation. If the signal propagation to the stations involves atmospheric reflections, the equations for the $D_{i}$ change and thus the estimator changes.

In general, the least squares estimator requires knowledge of the statistics of the measurement errors. However, if (85) applies, if the covariances of the $\epsilon_{i}$ are zero, and if the variances of the $\epsilon_{i}$ have the common value $\sigma_{t}^{2}$, then cancellation in (86) leaves an estimator that is independent of $\sigma_{t}^{2}$. Equality of the variances is a reasonable assumption for stations with identical receivers that are much closer to each other than to the transmitter.

Let $\sigma_{t i}^{2}$ denote the variance of the measured arrival time $t_{i}$ at station $i$. The mean-square ranging error is defined as $c^{2} \sigma_{s}^{2}$, where

$\sigma_{s}^{2}=\frac{1}{N} \sum_{i=1}^{N} \sigma_{t i}^{2}$

is the average variance of the arrival times. The geometric dilution of precision (GDOP) is defined as the ratio of the root-mean-square position error $\epsilon_{r}$ to the rootmean-square ranging error. It follows from (52) that the GDOP associated with an unbiased estimator and a hyperbolic system is

GDOP $=\sqrt{\operatorname{trace}[\boldsymbol{P}]} / c \sigma_{s}$.

The GDOP indicates how much the fundamental ranging error, intuitively measured by $c \sigma_{s}$, is magnified by the geometric relation among the transmitter position and the stations. If the geometry is such that the arrival-time variances are nearly equal, then the GDOP is only weakly dependent on them. For the two-dimensional location problem, (74) and (89) yield

$\mathrm{CEP} \approx\left(0.75 c \sigma_{s}\right) \mathrm{GDOP}$.

Since the arrival-time variance $\sigma_{t}^{2}$ is due primarily to the thermal and environmental noise, it is often reasonable to model $\epsilon_{i}$ as the sum of a constant bias plus zero-mean white Gaussian noise. The Cramer-Rao bound for an arrival-time estimate in the presence of white Gaussian noise gives [6]

$\sigma_{t}^{2} \geq\left[\left(2 E / N_{0}\right) \beta_{r}^{2}\right]^{-1}$

where $E$ is the energy in the received signal, $N_{0} / 2$ is the two-sided noise power spectral density, and $\beta_{r}^{2}$ is a function of the bandwidth of the signal. If $S(\omega)$ denotes the Fourier transform of the signal, then

$\beta_{r}^{2}=\frac{\int_{-\infty}^{\infty} \omega^{2}|S(\omega)|^{2} d \omega}{\int_{-\infty}^{\infty}|S(\omega)|^{2} d \omega}$.

If the received signal consists of pulses, then $E$ is the sum of the energies of the individual pulses. An approximate model for many radar signals is a series of pulses, each of which results from passing a truncated sinusoid with an ideal rectangular envelope of duration $T_{p}$ through an ideal rectangular bandpass filter of bandwidth $B$ centered at the sinusoidal frequency. For each pulse and for the entire radar signal, (92) yields

$\beta_{r}^{2} \approx 2 B / T_{p}, \quad B T_{p}>>1$.

In contrast, for a signal with a uniform Fourier transform over a bandwidth $B$, (92) gives

$\beta_{r}^{2}=\pi^{2} B^{2} / 3$.

This model might approximate a communications signal.

Let $T$ denote the total signal duration, $R_{s}=E / T$ denote the average signal power at the receiver, and $D$ denote the distance between the transmitter and the receiver. Over a large range of values of $D$, it is often possible to approximate $R_{s}$ by [7]

$R_{s}=K_{E} \exp (-\alpha D) / D^{n}$

where $\alpha, n$, and $K_{E}$ are independent of $D$, but may be functions of other parameters such as the transmitter power, antenna gains, antenna heights, and the signal frequency. For optical and millimeter-wave frequencies, 
accurate modeling requires $\alpha>0$, but we may usually set $\alpha=0$ at other frequencies. Inequality (91) and (95) relate $\sigma_{t}^{2}$ to $D$.

As an important special case, we consider a transmitter and three stations in the same plane so that only two position coordinates are to be estimated. The planar model is reasonable if a transmitter and stations are near the surface of the Earth and close enough that the curvature of the Earth's surface can be neglected. One of the stations is designated the master station, and the other two are called slave stations. Arrival-time measurements at the slave stations are sent to the master station, where the time differences and then the position estimate are computed.

We assume that the $\epsilon_{i}$ are uncorrelated random variables so that

$N_{\epsilon}=\left[\begin{array}{ccc}\sigma_{t 1}^{2} & 0 & 0 \\ 0 & \sigma_{t 2}^{2} & 0 \\ 0 & 0 & \sigma_{t 3}^{2}\end{array}\right]$.

The $\boldsymbol{H}$ matrix for $N=3$ is

$\boldsymbol{H}=\left[\begin{array}{rrr}1 & -1 & 0 \\ 0 & 1 & -1\end{array}\right]$.

Let $\phi_{0 i}$ denote the bearing angle from station $i$ at coordinates $\left(x_{i}, y_{i}\right)$ to the reference point at coordinates $\left(x_{0}, y_{0}\right)$, as illustrated in Fig. 6. Thus

$\phi_{0 i}=\tan ^{-1}\left(\frac{y_{0}-y_{i}}{x_{0}-x_{i}}\right), \quad i=1,2,3$.

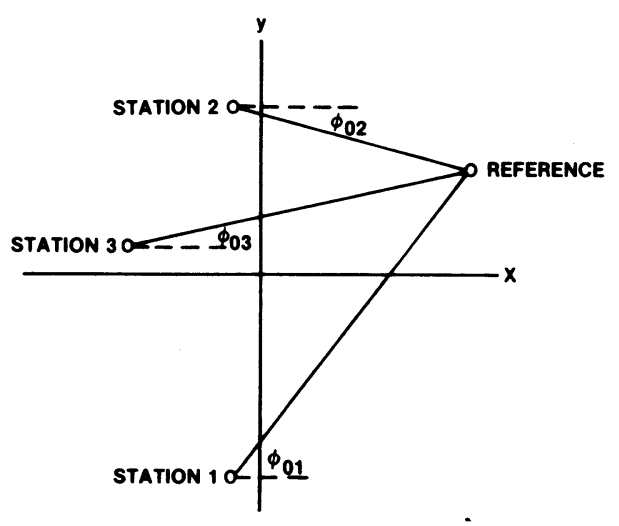

Fig. 6. Angle definitions for reference and three stations.

Equation (78) may be expressed as

$\boldsymbol{F}=\left[\begin{array}{cc}\cos \phi_{01} & \sin \phi_{01} \\ \cos \phi_{02} & \sin \phi_{02} \\ \cos \phi_{03} & \sin \phi_{03}\end{array}\right]$.

The covariance matrix $\boldsymbol{P}$ can be evaluated by substituting (85), (96), (97), and (99) into (87). The components of $\boldsymbol{P}$ defined by (53) are

$$
\begin{aligned}
\sigma_{1}^{2}= & \alpha\left[\sigma_{t 1}^{2}\left(\sin \phi_{02}-\sin \phi_{03}\right)^{2}\right. \\
& +\sigma_{t 2}^{2}\left(\sin \phi_{01}-\sin \phi_{03}\right)^{2} \\
& \left.+\sigma_{t 3}^{2}\left(\sin \phi_{01}-\sin \phi_{02}\right)^{2}\right]
\end{aligned}
$$

$$
\begin{aligned}
\sigma_{2}^{2}= & \alpha\left[\sigma_{t 1}^{2}\left(\cos \phi_{02}-\cos \phi_{03}\right)^{2}\right. \\
& +\sigma_{t 2}^{2}\left(\cos \phi_{01}-\cos \phi_{03}\right)^{2} \\
& \left.+\sigma_{t 3}^{2}\left(\cos \phi_{01}-\cos \phi_{02}\right)^{2}\right] \\
\sigma_{12}= & \alpha\left[\sigma_{t 1}^{2}\left(\cos \phi_{03}-\cos \phi_{02}\right)\right. \\
& \left(\sin \phi_{02}-\sin \phi_{03}\right) \\
& +\sigma_{t 2}^{2}\left(\cos \phi_{03}-\cos \phi_{01}\right) \\
& \left(\sin \phi_{01}-\sin \phi_{03}\right) \\
& +\sigma_{t 3}^{2}\left(\cos \phi_{02}-\cos \phi_{01}\right) \\
& \left.\left(\sin \phi_{01}-\sin \phi_{02}\right)\right]
\end{aligned}
$$

where

$$
\begin{aligned}
\alpha= & c^{2}\left[\left(\cos \phi_{01}-\cos \phi_{02}\right)\left(\sin \phi_{02}-\sin \phi_{03}\right)\right. \\
& -\left(\cos \phi_{02}-\cos \phi_{03}\right) \\
& \left.\left(\sin \phi_{01}-\sin \phi_{02}\right)\right]^{-2} .
\end{aligned}
$$

If any two bearing angles are equal, then $\sigma_{1}^{2}, \sigma_{2}^{2}$, and $\sigma_{12}$ $\rightarrow \infty$. These events correspond to reference points that lie along a line passing through two of the stations.

The least squares or maximum likelihood estimator, determined from (86), is

$$
\begin{aligned}
\hat{x}= & x_{0}+\sqrt{\alpha}\left[\left(t_{1}-D_{01} / c\right)\left(\sin \phi_{02}-\sin \phi_{03}\right)\right. \\
& +\left(t_{2}-D_{02} / c\right)\left(\sin \phi_{03}-\sin \phi_{01}\right) \\
& \left.+\left(t_{3}-D_{03} / c\right)\left(\sin \phi_{01}-\sin \phi_{02}\right)\right] \\
\hat{y}= & y_{0}+\sqrt{\alpha}\left[\left(t_{1}-D_{01} / c\right)\left(\cos \phi_{03}-\cos \phi_{02}\right)\right. \\
& +\left(t_{2}-D_{02} / c\right)\left(\cos \phi_{01}-\cos \phi_{03}\right) \\
& \left.+\left(t_{3}-D_{03} / c\right)\left(\cos \phi_{02}-\cos \phi_{01}\right)\right] .
\end{aligned}
$$

To determine the transmitter range, which may be defined as the distance between the transmitter and the master station, it is convenient to align the $x$ axis with the line between the master station and the reference point and to place the origin of the coordinate system at the master station. If the reference point is near the transmitter position, then $\hat{x}$ is a suitable range estimator and $\sigma_{1}^{2}$ approximates the variance of the range estimator; otherwise, the range can be estimated by $\left(\hat{x}^{2}+\hat{y}^{2}\right)^{1 / 2}$. A suitable estimator for the bearing with respect to the $x$ axis is

$\hat{\phi}=\tan ^{-1}(\hat{y} / \hat{x})$.

The estimator bias can be determined from (15). Neglecting the linearization error and using (83), we obtain

$$
\begin{aligned}
b_{1}= & \sqrt{\alpha}\left\{E\left[\epsilon_{1}\right]\left(\sin \phi_{02}-\sin \phi_{03}\right)\right. \\
& +E\left[\epsilon_{2}\right]\left(\sin \phi_{03}-\sin \phi_{01}\right) \\
& \left.+E\left[\epsilon_{3}\right]\left(\sin \phi_{01}-\sin \phi_{02}\right)\right\}
\end{aligned}
$$




$$
\begin{aligned}
b_{2}= & \sqrt{\alpha}\left\{E\left[\epsilon_{1}\right]\left(\cos \phi_{03}-\cos \phi_{02}\right)\right. \\
& +E\left[\epsilon_{2}\right]\left(\cos \phi_{01}-\cos \phi_{03}\right) \\
& \left.+E\left[\epsilon_{3}\right]\left(\cos \phi_{02}-\cos \phi_{01}\right)\right\} .
\end{aligned}
$$

Nonzero values of the $E\left[\epsilon_{i}\right]$ are caused primarily by uncertainties in the station positions, synchronization errors, and the temperature dependence of the receiver delays and filter characteristics.

Assuming that $\boldsymbol{n}=\boldsymbol{H} \boldsymbol{\epsilon}$ has a Gaussian distribution, (54), (55), (73), and (100)-(103) give the CEP in terms of the bearing angles and the arrival-time variances. For a fixed deployment of stations, the locus of transmitter positions with a constant value of the CEP can be determined numerically. For this purpose, the equations may be expressed in terms of the Cartesian coordinates by using (98) and it is assumed that the reference point coincides with the transmitter position with negligible error so that $D_{0 i}=D D_{i}$.

Let $L$ denote the length of a linear array of three stations with coordinates $(0,-L / 2),(0,0)$, and $(0, L / 2)$. Assuming that the lower bound of inequality (91) is nearly achieved and using (95), we obtain

$$
\begin{aligned}
\sigma_{t i}^{2} & \simeq \sigma_{t L}^{2}\left(D_{0 i} / L\right)^{n} \exp \left[\alpha\left(D_{0 i}-L\right)\right], \quad i=1,2,3 \\
\sigma_{t L}^{2} & =N_{0} L^{n} \exp (\alpha L) / 2 \beta_{r}^{2} T K_{E}
\end{aligned}
$$

where $\sigma_{t L}$ denotes the lower bound of $\sigma_{t i}$ when $D_{0 i}=L$. It is assumed that $\alpha, n, K_{E}$, and hence $\sigma_{t L}$ are identical for all three stations. We assume that the transmitter and the stations have omnidirectional antennas so that $K_{E}$ does not depend upon the bearing angle to the transmitter. Figs. 7 and 8 depict loci of constant values of $\mathrm{CEP} / c \sigma_{t L}$

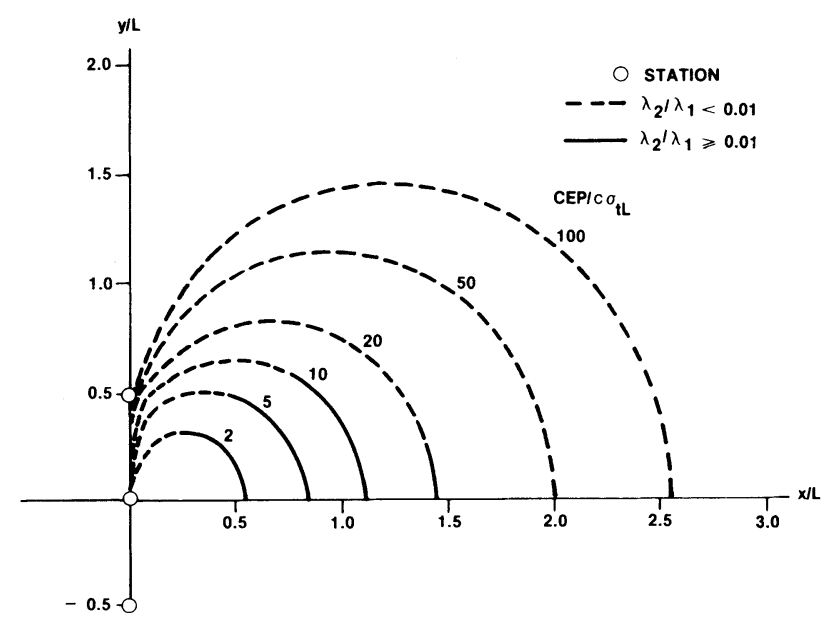

Fig. 7. Loci of constant CEP/c $\sigma_{t L}$ for linear array of three stations with $n=2$.

for $\alpha=0$. Only the first quadrant is displayed because of the symmetry of the loci. Fig. 7 assumes $n=2$, which corresponds to free-space propagation. Fig. 8 assumes $n=4$, which might model VHF propagation near the Earth's surface.

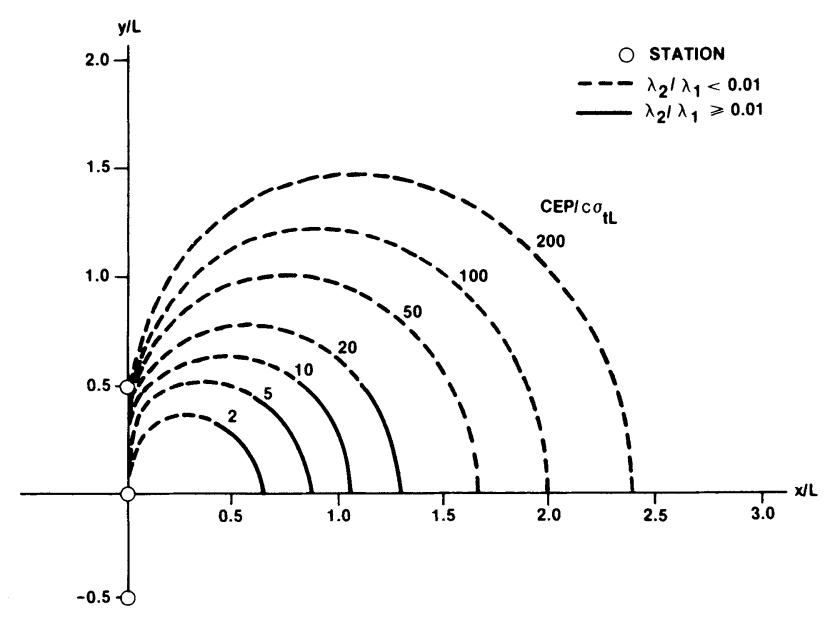

Fig. 8. Loci of constant $\mathrm{CEP} / c \sigma_{t L}$ for linear array of three stations with $n=4$.

In Fig. 9, the stations form a nonlinear array with coordinates $(0,-L / 2),(-L / 2,0)$, and $0, L / 2)$, respectively. The most significant features are the

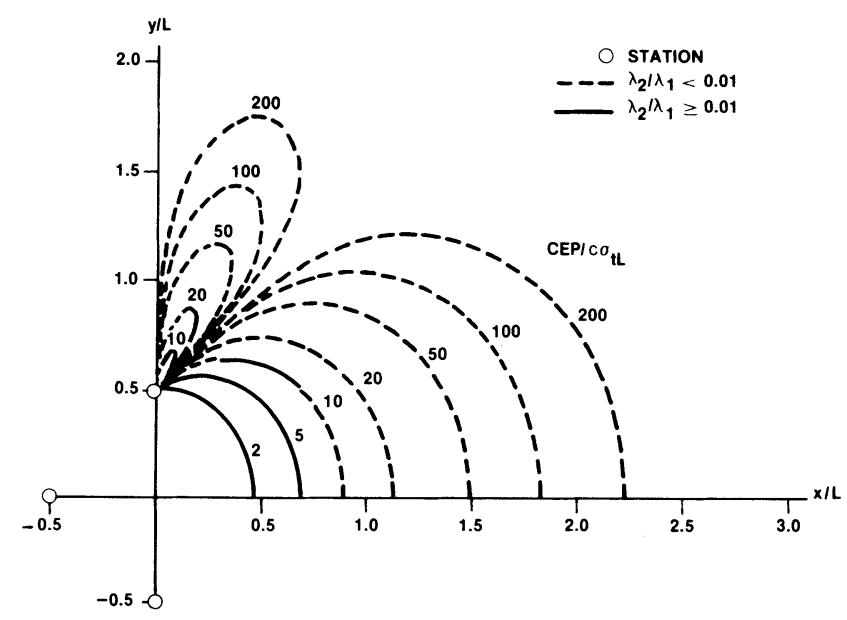

Fig. 9. Loci of constant $\mathrm{CEP} / c \sigma_{t L}$ for nonlinear array of three stations with $n=4$.

singularities in the values of the CEP along the lines passing through two of the stations. Consequently, only a slight spatial nonlinearity is permissible if a broad field of view is required. However, other important factors in the choice of station positions are the needs to maintain lineof-sight paths from potential transmitter positions and to minimize the potential multipath interference.

In Figs. 7-9, the parts of the loci for which $\lambda_{2}<$ $0.01 \lambda_{1}$ are indicated by dotted lines. For these small values of $\lambda_{2} / \lambda_{1}$, the CEP is a questionable measure of performance of the passive location system. A more suitable measure may be the length of the major axis of the concentration ellipse,

$L_{e}=2 \sqrt{\kappa \lambda_{1}}$.

It follows from (73) that

$\mathrm{CEP} \approx 0.563 L_{e} / 2 \sqrt{\kappa}, \quad \lambda_{2}<0.01 \lambda_{1}$ 
where $\kappa$ is given by $(60)$. Thus the dotted lines approximate the loci of constant values of $L_{e} / 3.552 \sqrt{\kappa} c \sigma_{t L}$.

\section{LOCATION USING BEARING MEASUREMENTS}

The bearing measurements of passive direction-finding systems at two or more stations or points along an aircraft trajectory can be combined by a direction-finding location system to produce an estimate of transmitter position. The transmitted signal may be received at a station by line-ofsight propagation or after atmospheric reflection at a known altitude. A single bearing angle may be measured at each station of the location system. Alternatively, separate azimuth and elevation angle measurements, possibly made by orthogonal interferometers, can be used to determine transmitter position. In the absence of noise and interference, bearing lines from two or more stations will intersect to determine a unique location. In the presence of noise, more than two bearing lines will not intersect at a single point, as illustrated for a planar configuration in Fig. 10. Consequently, processing is

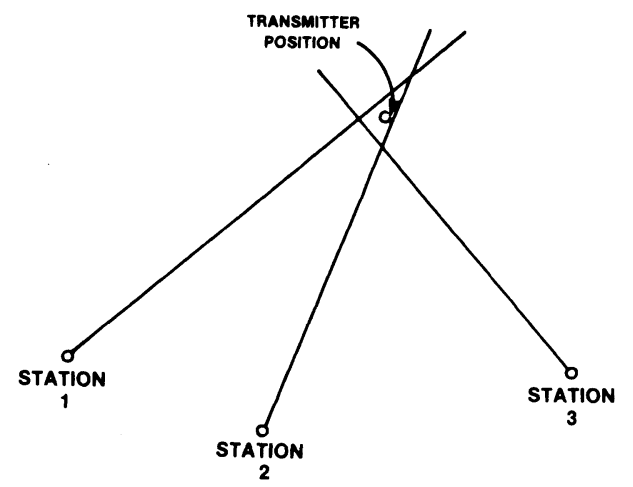

Fig. 10. Bearing lines from three direction-finding systems.

required to determine the optimal position estimate. Let $\theta_{i}$ denote the bearing angle measured at station $i$ relative to a baseline in a three-dimensional coordinate system defined so that the $x$ axis is parallel to the baseline, as shown in Fig. 11. If the coordinates of the station are

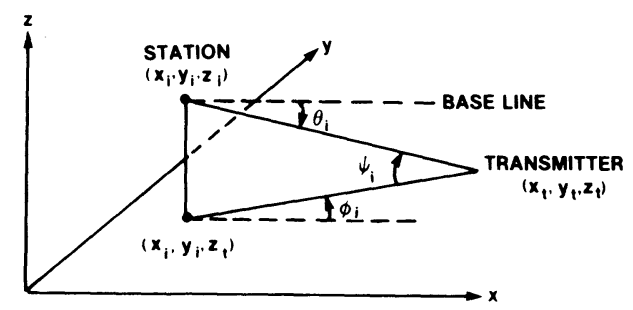

Fig. 11. Angle definitions for direction-finding systems.

$\left(x_{i}, y_{i}, z_{i}\right)$ and the coordinates of the transmitter are $\left(x_{t}, y_{t}, z_{t}\right)$, then in the absence of measurement errors, line-of-sight propagation implies that

$$
\begin{gathered}
\theta_{i}=\cos ^{-1}\left[\frac{x_{t}-x_{i}}{\sqrt{\left(x_{t}-x_{i}\right)^{2}+\left(y_{t}-y_{i}\right)^{2}+\left(z_{t}-z_{i}\right)^{2}}}\right], \\
0 \leq \theta_{i} \leq \pi
\end{gathered}
$$

In Fig. 11, the azimuth angle $\phi_{i}$ is defined in the plane passing through the transmitter and perpendisular to the $z$ axis. It is positive in the counterclockwise direction relative to the positive $x$ axis. If the elevation angle $\psi_{i}$ of the station relative to the transmitter is known approximately or is estimated by a suitable means, such as a vertical interferometer, then $\phi_{i}$ may be calculated using the geometrical relation

$\cos \theta_{i}=\cos \phi_{i} \cos \psi_{i}$

which is easily derived from Fig. 11. If $\psi_{i}$ is sufficiently small, the measured bearing is well approximated by the azimuth, which is defined by

$\phi_{i}=\tan ^{-1}\left(\frac{y_{t}-y_{i}}{x_{t}-x_{i}}\right)$.

In most applications, the transmitter is known to lie on the surface of the Earth or at a fixed altitude so that $z_{t}$ is known and does not have to be estimated. Equation (115) is used in the estimation of the $\left(x_{t}, y_{t}\right)$. The use of this equation is equivalent to the representation of the threedimensional problem by a two-dimensional model. In the model, the transmitter and the stations are assumed to lie in the same plane so that the azimuths are identical to the bearings. If the transmitter and the stations actually lie on the Earth's surface, the model is an idealization that neglects the curvature of the surface. Two-dimensional position estimation using bearing information is often called triangulation.

We consider in detail the estimation of the twodimensional column vector $\boldsymbol{R}$ having components $x$ and $y$. Line-of-sight propagation is assumed. The measured bearing angle $\phi_{i}$ and the measurement error $n_{i}$ satisfy

$\phi_{i}=f_{i}(\boldsymbol{R})+n_{i}, \quad i=1,2, \ldots, N$

where

$f_{i}(\boldsymbol{R})=\tan ^{-1}\left(\frac{y-y_{i}}{x-x_{i}}\right), \quad i=1,2, \ldots, N$

and the station coordinates are $x_{i}$ and $y_{i}$. In matrix form, we have

$\phi=f(R)+n$.

Let the column vector $\boldsymbol{R}_{0}$ with components $x_{0}$ and $y_{0}$ specify a reference point, which may be chosen to be in the middle of the polygon bounded by the measured bearing lines. Let $\phi_{0 i}$ denote the bearing angle from station $i$ to the reference point. Then

$$
\begin{aligned}
\sin \phi_{0 i}=\frac{y_{0}-y_{i}}{D_{0 i}} ; \quad \cos \phi_{0 i} & =\frac{x_{0}-x_{i}}{D_{0 i}}, \\
& i=1,2, \ldots, N
\end{aligned}
$$

where

$$
\begin{aligned}
\mathrm{D}_{0 i}=\left[\left(x_{0}-x_{i}\right)^{2}+\left(y_{0}-y_{i}\right)^{2}\right]^{1 / 2} & \\
i & =1,2, \ldots, N .
\end{aligned}
$$


From (7) with $\boldsymbol{x}=\boldsymbol{R}$ and $\boldsymbol{x}_{0}=\boldsymbol{R}_{0}$, we obtain

$\boldsymbol{G}=\left[\begin{array}{cc}-\left(\sin \phi_{01}\right) / D_{01} & \left(\cos \phi_{01}\right) / D_{01} \\ \vdots & \vdots \\ -\left(\sin \phi_{0 N}\right) / D_{0 N} & \left(\cos \phi_{0 N}\right) / D_{0 N}\end{array}\right]$.

The least squares or maximum likelihood estimator is

$\hat{\boldsymbol{R}}=\boldsymbol{R}_{0}+\left(\boldsymbol{G}^{\mathrm{T}} \boldsymbol{N}^{-1} \boldsymbol{G}\right)^{-1} \boldsymbol{G}^{\mathrm{T}} \boldsymbol{N}^{-1} \boldsymbol{\phi}_{r}$

where $N$ is the covariance matrix of the bearing measurement errors and

$\boldsymbol{\phi}_{r}=\boldsymbol{\phi}-\boldsymbol{f}\left(\boldsymbol{R}_{0}\right)$.

The $i$ th component of $\boldsymbol{\phi}_{r}$ is

$$
\begin{array}{r}
\phi_{r i}=\phi_{i}-\phi_{0 i}=\phi_{i}-\tan ^{-1}\left(\frac{y_{0}-y_{i}}{x_{0}-x_{i}}\right), \\
i=1,2, \ldots, N
\end{array}
$$

which is the bearing angle relative to the line between station $i$ and the reference point, as depicted in Fig. 12.

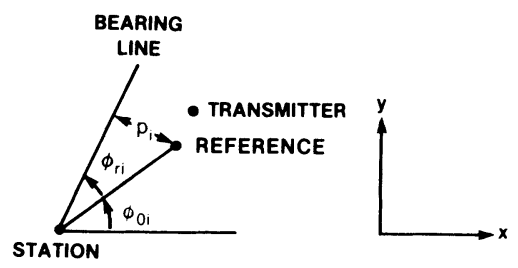

Fig. 12. Geometry of transmitter, reference point, and station.

If the bearing measurement errors are independent random variables with variances $\sigma_{\phi i}^{2}, i=1,2, \ldots, N$, then

$\boldsymbol{N}=\left[\begin{array}{ccc}\sigma_{\phi 1}^{2} & & 0 \\ & \ddots & \\ 0 & & \sigma_{\phi N}^{2}\end{array}\right]$.

Direct calculation using (16), (121), and (125) establishes that the elements of the covariance matrix of $\hat{\boldsymbol{R}}$ are

$$
\begin{aligned}
\sigma_{1}^{2} & =E\left[(\hat{x}-x)^{2}\right]=\frac{\mu}{\mu \lambda-v^{2}} \\
\sigma_{2}^{2} & =E\left[(\hat{y}-y)^{2}\right]=\frac{\lambda}{\mu \lambda-v^{2}} \\
\sigma_{12} & =E[(\hat{x}-x)(\hat{y}-y)]=\frac{v}{\mu \lambda-v^{2}}
\end{aligned}
$$

where

$$
\begin{aligned}
\mu & =\sum_{i=1}^{N} \frac{\cos ^{2} \phi_{0 i}}{D_{0 i}^{2} \sigma_{\phi i}^{2}} \\
\lambda & =\sum_{i=1}^{N} \frac{\sin ^{2} \phi_{0 i}}{D_{0 i}^{2} \sigma_{\phi i}^{2}} \\
\nu & =\sum_{i=1}^{N} \frac{\sin \phi_{0 i} \cos \phi_{0 i}}{D_{0 i}^{2} \sigma_{\phi i}^{2}} .
\end{aligned}
$$

It follows from (121), (122), and (125) that the components of the linearized least squares estimator are

$$
\begin{aligned}
& \hat{x}= x_{0}+\frac{1}{\mu \lambda-v^{2}} \sum_{i=1}^{N} \\
& \phi_{r i} \frac{\left(\nu \cos \phi_{0 i}-\mu \sin \phi_{0 i}\right)}{D_{0 i} \sigma_{\phi i}^{2}} \\
& \hat{y}=y_{0}+\frac{1}{\mu \lambda-v^{2}} \sum_{i=1}^{N} \\
& \phi_{r i} \frac{\left(\lambda \cos \phi_{0 i}-v \sin \phi_{0 i}\right) .}{D_{0 i} \sigma_{\phi i}^{2}}
\end{aligned}
$$

Similarly, if the linearization error is negligible, the bias components are

$$
\begin{aligned}
b_{1}= & \frac{1}{\mu \lambda-v^{2}} \sum_{i=1}^{N} \\
& E\left[n_{i}\right] \frac{\left(\nu \cos \phi_{0 i}-\mu \sin \phi_{0 i}\right)}{D_{0 i} \sigma_{\phi i}^{2}} \\
b_{2}= & \frac{1}{\mu \lambda-v^{2}} \sum_{i=I}^{N} \\
& E\left[n_{i}\right] \frac{\left(\lambda \cos \phi_{0 i}-v \sin \phi_{0 i}\right)}{D_{0 i} \sigma_{\phi i}^{2}} .
\end{aligned}
$$

The dependence of the estimator and bias on $\sigma_{\phi i}^{2}, i=$ $1,2, \ldots, N$, is eliminated because of cancellation in (132) to (135) if these variances are all equal. This equality is a reasonable assumption if the receivers are identical and much closer to each other than to the transmitter.

Let $p_{i}$ denote the shortest distance from the reference point to the measured bearing line at station $i$, as depicted in Fig. 12. Suppose that the reference point is close to the true transmitter position and that the measurement errors are small. Then

$$
\phi_{r i} \approx p_{i} / D_{0 i}, \quad i=1,2, \ldots, N
$$

$\cos \phi_{0 i} \approx \cos \phi_{i} ; \quad \sin \phi_{0 i} \approx \sin \phi_{i}$,

$$
i=1,2, \ldots, N \text {. }
$$

Substituting (136) and (137) into (129) to (133), we obtain the components of an estimator that depends upon the measurements $p_{i}$ and $\phi_{i}, i=1,2, \ldots, N$. This estimator, called the Stansfield algorithm, was originally derived from heuristic arguments and the assumption of small bearing measurement errors [8]. If $\boldsymbol{R}_{0}$ is close to $\boldsymbol{R}$, then the linearized least squares estimator is preferable to the Stansfield algorithm, which produces a larger estimator bias unless the bearing errors are small. However, if the bearing errors are large, it may not be possible to choose $\boldsymbol{R}_{0}$ close to $\boldsymbol{R}$. In this case, it is not clear which estimator is preferable.

The mean-square ranging error associated with direction-finding systems is defined as the average variance of $D_{0 i} \phi_{r i}$ : 
$\sigma_{d}^{2}=\frac{1}{N} \sum_{i=1}^{N} D_{0 i}^{2} \sigma_{\phi i}^{2}$.

In analogy to (89), the GDOP associated with an unbiased estimator and a direction-finding location system is defined as

GDOP $=\sqrt{\operatorname{trace}[\boldsymbol{P}]} / \sigma_{d}$.

If the geometry is such that the bearing variances are nearly equal, then the GDOP is only weakly dependent on them. From (74), it follows that

$\mathrm{CEP} \approx\left(0.75 \sigma_{d}\right) \mathrm{GDOP}$.

The variance of a bearing estimator $\sigma_{\phi}^{2}$ is due primarily to the thermal and environmental noise. Approximate expressions for $\sigma_{\phi}^{2}$ are known for various direction-finding systems operating in white Gaussian noise [7]. In most cases, if $E / N_{0}$ is sufficiently large, $\sigma_{\phi}^{2}$ can be expressed in the form

$$
\sigma_{\phi}^{2} \simeq\left(\frac{2 E}{N_{0}} \beta_{\phi}^{2}\right)^{-1}
$$

where $\beta_{\phi}^{2}$ is a function of the system parameters other than $E / N_{0}$, and the variation of the signal energy with the distance to the transmitter can be determined from (95).

For example, consider a planar configuration and a phase interferometer with its antennas pointing in the direction of the positive $x$ axis. It can be shown that if the estimator bias is negligible, then [7]

$\sigma_{\phi}^{2} \geq\left(\frac{c}{2 \pi f_{0} d \cos \phi}\right)^{2}\left(\frac{E}{N_{0}}\right)^{-1}, \quad|\phi|<\frac{\pi}{2}$

where $f_{0}$ is the carrier frequency of the received signal, $d$ is the maximum separation between the interferometer antennas, and $\phi$ is the true bearing angle.

As a specific example, we consider identical stations that are symmetrically located with respect to the reference point so that

$$
\begin{aligned}
\phi_{0 i}= & -\phi_{0(N-i+1)}, \quad i=1,2, \ldots,[N / 2] \\
D_{0 i}^{2} \sigma_{\phi i}^{2}= & D_{0(N-i+1)}^{2} \sigma_{\phi(N-i+1)}^{2}, \\
& i=1,2, \ldots,[N / 2]
\end{aligned}
$$

where $[x]$ denotes the largest integer in $x$. If $N$ is odd, we further assume that

$\phi_{0 i}=0, \quad i=[N / 2]+1 ; N$ is odd.

A possible configuration for $N=5$ is illustrated in Fig. 13. This example is probably unrealistic for ground stations if $N \geq 4$, but might adequately represent location estimation by an aircraft that samples bearing data at evenly spaced points along its trajectory. Substitution of (143), (144), and (145) into (131) yields $v=0$, which implies that $\sigma_{12}=0$. We conclude that the symmetrical, but not necessarily linear, placement of the stations with respect to an accurately located reference leads to uncorrelated coordinate estimates. For an aircraft, we

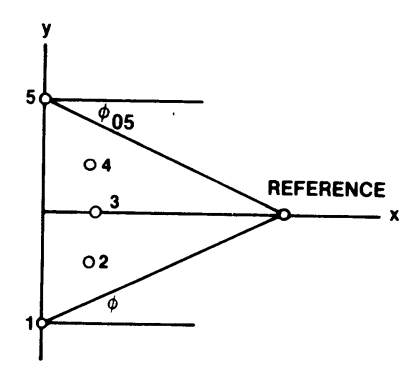

Fig. 13. Configuration of five symmetrically located stations.

may interpret $\sigma_{2}^{2}$ as the variance of the "cross-range" estimation error and $\sigma_{1}^{2}$ as the variance of the "downrange" estimation error.

If $N=2$, then (126) to (133) give

$\sigma_{1}^{2}=\frac{D_{01}^{2} \sigma_{\phi 1}^{2}}{2 \sin ^{2} \phi_{01}}$

$\sigma_{2}^{2}=\frac{D_{01}^{2} \sigma_{\phi 1}^{2}}{2 \cos ^{2} \phi_{01}}$

$$
\hat{x}=x_{0}-\frac{D_{01}}{2 \sin \phi_{01}}\left(\phi_{r 1}-\phi_{r 2}\right)
$$

$$
\hat{y}=y_{0}+\frac{D_{01}}{2 \cos \phi_{01}}\left(\phi_{r 1}+\phi_{r 2}\right)
$$

If the reference point is located at the intersection of the two measured bearing lines, then $\phi_{r 1}=\phi_{r 2}=0$. It follows that $(\hat{x}, \hat{y})=\left(x_{0}, y_{0}\right)$, as expected. From (138), (139), (146), and (147), we obtain

$\mathrm{GDOP}=\frac{\sqrt{2}}{\sin 2 \phi_{01}}$.

The minimum value of the GDOP, equal to $\sqrt{2}$, is attained when $\phi_{01}=\pi / 4$. Since $\sigma_{12}=0,(54),(55)$, and (73) give

$$
\mathrm{CEP}=0.563 \max \left(\sigma_{1}, \sigma_{2}\right)+0.614 \min \left(\sigma_{1}, \sigma_{2}\right) \text {. }
$$

If $N=3$, the variance of $\hat{x}$ remains the same, but the variance of $\hat{y}$ becomes

$\sigma_{2}^{2}=\left(\frac{2 \cos ^{2} \phi_{01}}{D_{01}^{2} \sigma_{\phi 1}^{2}}+\frac{1}{D_{02}^{2} \sigma_{\phi 2}^{2}}\right)^{-1}$

which shows that the extra station only improves the estimation of the $y$ coordinate of the transmitter. As the transmitter range increases, $\phi_{01}$ decreases and thus $\sigma_{1}^{2} / \sigma_{2}^{2}$ increases.

If $\boldsymbol{n}$ in (118) has a Gaussian distribution, then (54), (55), (73), and (126)-(131) give the CEP in terms of the bearing angles and their variances. Assuming that the reference point coincides with the transmitter position so that $D_{0 i}=D_{i}$ and $\phi_{0 i}$ is equal to the bearing angle to the transmitter position, the locus of positions with a constant CEP can be determined numerically by using (119) and (120). 
Consider a linear array of three stations with coordinates $(0,-L / 2),(0,0)$, and $(0, L / 2)$. Each station has an interferometer with omnidirectional antennas pointing in the direction of the positive $x$ axis. Let $\sigma_{\phi L}$ denote the value of $\sigma_{\phi i}$ when $D_{0 i}=L$ and $\phi_{0 i}=0$. Assuming that $\sigma_{\phi L}, n$, and $\alpha$ are identical for all three stations and that the lower bound of inequality (142) is nearly achieved, (95) yields

$$
\begin{aligned}
\sigma_{\phi i}^{2} \simeq & \frac{\sigma_{\phi L}^{2}}{\cos ^{2} \phi_{0 i}}\left(\frac{D_{0 i}}{L}\right)^{n} \exp \left[\alpha\left(D_{0 i}-L\right)\right], \\
& \left|\phi_{i}\right|<\frac{\pi}{2} ; i=1,2,3 \\
\sigma_{\phi L}^{2}= & \frac{c^{2} N_{0} L^{n} \exp (\alpha L)}{\left(2 \pi f_{0} d\right)^{2} T K_{E}} .
\end{aligned}
$$

Figs. 14 and 15 depict loci of constant values of CEP/ $L \sigma_{\phi L}$ for $\alpha=0$. The loci are similar in form to those for

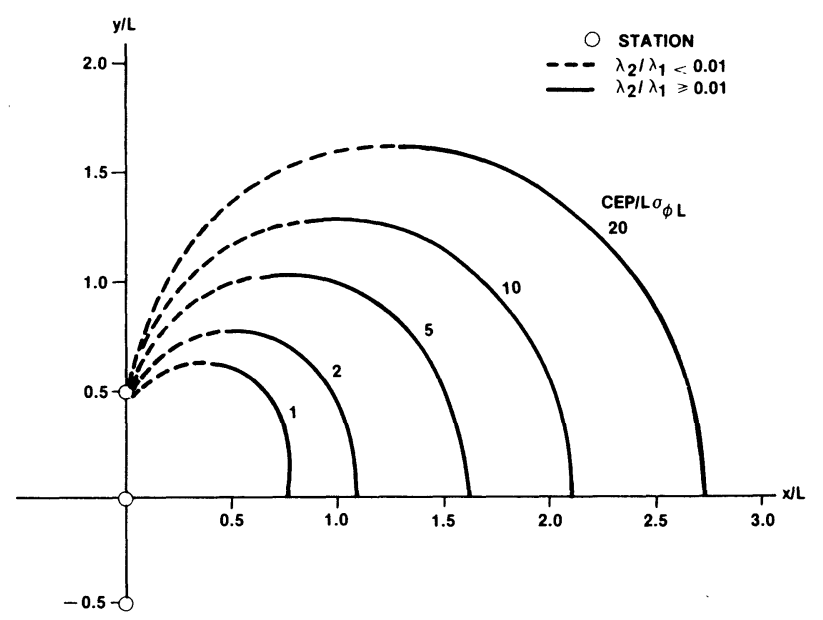

Fig. 14. Loci of constant $C E P / L \sigma_{\phi L}$ for linear array of three stations with $n=2$.

hyperbolic location systems. From (112), it follows that the dotted lines approximate the loci of constant values of $L_{e} / 3.552 \sqrt{\kappa} L \sigma_{\phi L}$. At equal distances from the array, direction-finding location systems produce less eccentric

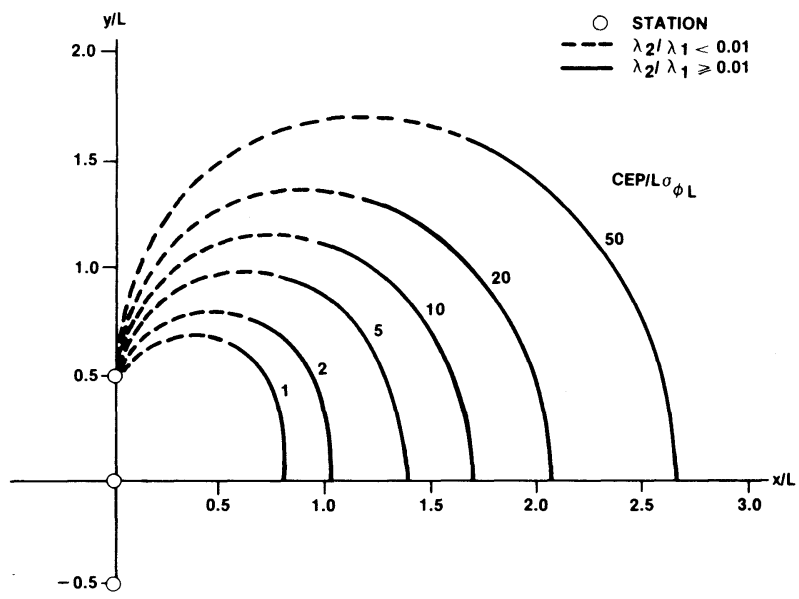

Fig. 15. Loci of constant CEP/L $\sigma_{\phi L}$ for linear array of three stations with $n=4$. concentration ellipses than similarly deployed hyperbolic location systems. This feature may be a significant factor in selecting the appropriate location systems for specific applications.

In Fig. 16, the stations form a nonlinear array with coordinates $(0,-L / 2),(-L / 2,0)$, and $(0, L / 2)$. A comparison with Fig. 9 indicates that the adverse effect of the nonlinear configuration is usually less for directionfinding systems than for hyperbolic systems.

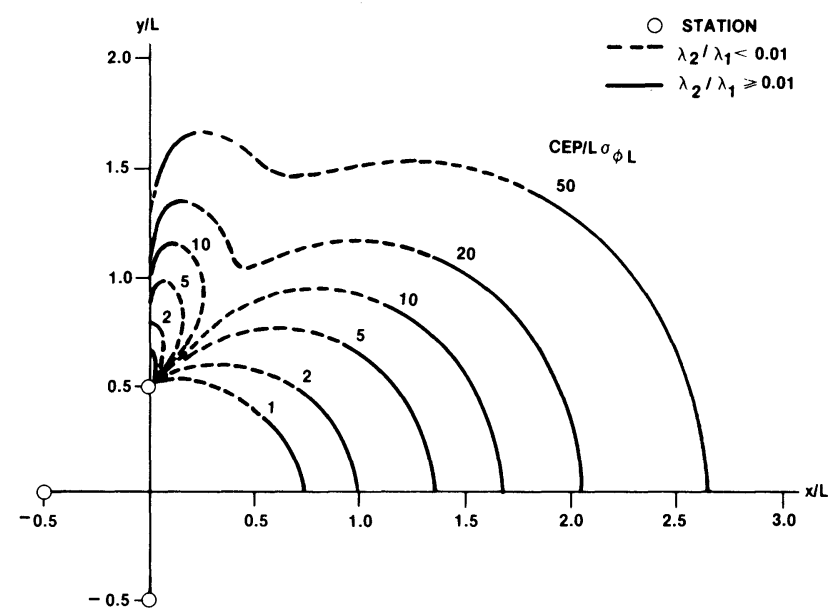

Fig. 16. Loci of constant CEP/L $\sigma_{\phi L}$ for nonlinear array of three stations with $n=4$.

Fig. 17 plots the constant $C E P / L \sigma_{\phi L}$ loci for a linear array of five stations with coordinates $(0,-L / 2),(0$, $-L / 4),(0,0),(0, l / 4)$, and $(0, L / 2)$. A comparison with Fig. 15 shows the CEP improvement from adding two stations while maintaining a constant baseline length equal to $L$. In general, the CEP is roughly inversely proportional to $\sqrt{N}$.

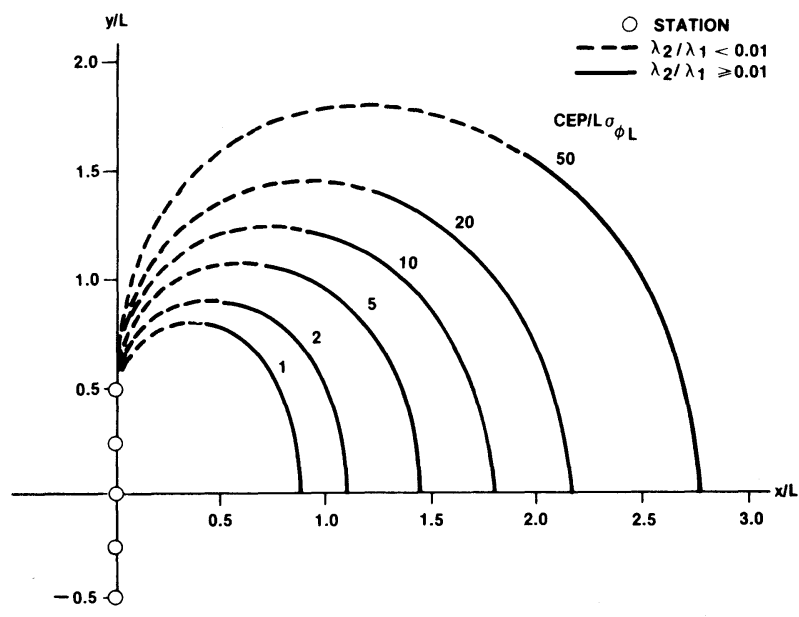

Fig. 17. Loci of constant $C E P / L \sigma_{\phi L}$ for linear array of five stations with $n=4$.

For a two-dimensional transmitter location with three stations, a comparison of Figs. 14-16 with Figs. 7-9 indicates that for hyperbolic systems to offer a significant performance advantage over direction-finding location systems when $\alpha=0$, it is necessary that 
$q c \sigma_{t L}<L \sigma_{\phi L}$

where $q \approx 5$. Substituting (154) and (110) and assuming equal parameter values for the two systems, we obtain the criterion

$\sqrt{2} \pi q f_{0} d<L \beta_{r}$.

Consequently, for the radar signal leading to (93), hyperbolic systems offer a potential advantage only if

$T_{p}\left(\pi q f_{0} d\right)^{2}<B L^{2}$.

For the communications signal leading to (94), a significant advantage requires

$\sqrt{6} q f_{0} d<B L$.

Inequalities (157) and (158) indicate that hyperbolic systems increase in desirability as the array length and signal bandwidth increase.

\section{OTHER LOCATION METHODS}

When the receivers are moving, it may be possible to use the known receiver trajectories to enhance the accuracy of the transmitter location. For example, three bearing measurements and two turns by an aircraft can be used to greatly reduce the effect of strong unknown biases in the measurements [9].

Moving receivers can exploit the Doppler shift in several ways. In the absence of noise, the measured frequency at a receiver $f_{m}$ is related to the transmitted frequency $f_{t}$ by

$f_{m}=f_{t}+f_{t} v_{r} / c=f_{t}+f_{t} v \cos \phi / c$

where $c$ is the signal velocity, $v_{r}$ is the velocity of the receiver in the direction to the transmitter, $v$ is the receiver velocity, and $\phi$ is the bearing angle to the transmitter relative to the velocity vector, as shown in Fig. 18.

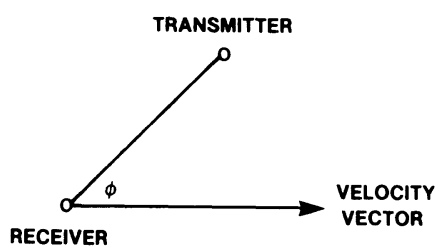

Fig. 18. Moving receiver.

Therefore, the bearing angle can be estimated if $f_{m}$ is measured and $f_{t}, v$, and $c$ are known. Bearing measurements from several receivers can be combined to obtain a transmitter location estimate, as is done in
Section VI. Another approach, which may be less sensitive to inaccuracies in the assumed value of $f_{t}$, is to measure the Doppler difference, which is defined as

$f_{m 1}-f_{m 2}=\left(f_{t} / c\right)\left(v_{1} \cos \phi_{1}-v_{2} \cos \phi_{2}\right)$

where the subscripts 1 and 2 refer to receivers 1 and 2 . The differential Doppler is defined as the integral of $f_{m 1}$ $-f_{m 2}$ over time. If $f_{t}$ does not change too rapidly over the integration interval, the differential Doppler is

$$
\begin{aligned}
\int_{t_{1}}^{t_{2}}\left(f_{m 1}-f_{m 2}\right) d t \simeq\left(f_{t a} / c\right)[ & D_{1}\left(t_{2}\right)-D_{1}\left(t_{1}\right) \\
- & \left.D_{2}\left(t_{2}\right)+D_{2}\left(t_{1}\right)\right]
\end{aligned}
$$

where $f_{t a}$ is the average transmitted frequency and $D_{i}\left(t_{j}\right)$, $i, j=1,2$, is the distance of receiver $i$ from the transmitter at time $j$. The right-hand sides of (160) and (161) can be expressed in terms of the transmitter coordinates. Thus, in the absence of noise, a Doppler difference or a differential Doppler measurement determines a surface on which the transmitter must lie. A location estimator can be derived in a manner analogous to the derivations of Sections V and VI. Because of the need for a precise estimate of $f_{t}$ or $f_{t a}$, Doppler location systems appear to be most useful in the location of transmitters of narrowband signals.

Doppler, arrival-time, and bearing measurements at the same or different receivers can be combined in hybrid location systems. The combined measurements may allow a reduction in the number of receivers required for a given location accuracy and may facilitate the resolution of ambiguities.

To accommodate a moving transmitter, the observation interval can be decreased so that the transmitter is nearly stationary during the interval and points on the trajectory can be located. However, decreases in the observation interval eventually lead to unacceptably large estimation errors, and other methods must be adopted. If the trajectory can be described by a low-order polynomial in time and if a sufficient number of stations or measurements are available, it is possible to estimate the coefficients by expanding the dimension of the estimator $\hat{\boldsymbol{x}}$. Alternatively, if the differential equations of motion are known, Kalman filters can be used to track the transmitter movement $[10,11]$. However, the implementation complexity of a passive location system with Kalman filters is usually considerably greater than that of a hyperbolic or direction-finding location system for stationary transmitters. 
[1] Wegner, L.H. (1971)

On the accuracy analysis of airborne techniques for passively locating electromagnetic emitters.

Report R-722-PR, Rand Corp.; available from National

Technical Information Service as ASTIA D.C. AD 729767 , 1971 .

[2] Lee, H.B. (1975)

A novel procedure for assessing the accuracy of hyperbolic multilateration systems.

IEEE Trans. Aerosp. Electron. Syst., AES-11 (Jan. 1975), 2.

[3] Sage, A.P., and Melsa, J.L. (1971)

Estimation Theory with Applications to Communications and Control.

New York: McGraw-Hill, 1971

[4] Butterly, P.J. (1972)

Position finding with empirical prior knowledge.

IEEE Trans. Aerosp. Electron. Syst., AES-8 (Mar. 1972), 142.

[5] Billingsley, P. (1979)

Probability and Measure.

New York: Wiley, 1979.
[6] Whalen, A.D. (1971)

Detection of Signals in Noise.

New York: Academic Press, 1971.

[7] Torrieri, D.J. (1981)

Principles of Military Communication Systems.

Dedham, Mass.: Artech House, 1981.

[8] Ancker, C.J. (1958)

Airborne direction finding-The theory of navigation errors. IRE Trans. Aeronaut. Navig. Electron., ANE-5 (Dec. 1958), 199.

[9] Mangel, M. (1981)

Three bearing method for passive triangulation in systems with unknown deterministic biases.

IEEE Trans. Aerosp. Electron. Syst., AES-17 (Nov. 1981), 814.

[10] Gelb, A. (1974) Applied Optimal Estimation.

Cambridge, Mass.: MIT Press 1974.

[11] Maybeck, P.S. (1979)

Stochastic Modeis, Estimation, and Control, vol. 1.

New York: Academic Press, 1979.

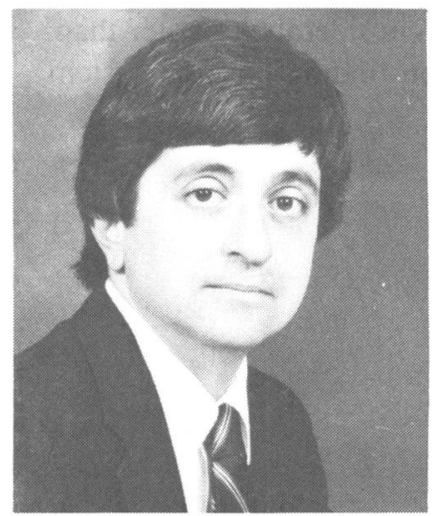

Don J. Torrieri received the B.S. degree from Massachusetts Institute of Technology, Cambridge, the M.S. degree from the Polytechnic Institute of New York, Brooklyn, and the Ph.D. degree in electrophysics from the University of Maryland, College Park.

He has worked on electronic systems at the Naval Research Laboratory, and since 1977 has been employed by the U.S. Army Countermeasures/Counter-

Countermeasures Center, Adelphi, Md. His primary interests are the analysis and assessment of communication systems and electronic countermeasures. He is the author of Principles of Military Communication Systems (Artech House, 1981) and a contributor to Acousto-Optic Signal Processing (N.J. Berg and J.N. Lee (Eds.), Marcel Dekker, 1983). 